Adv. Appl. Prob. 45, 981-1010 (2013)

Printed in Northern Ireland

(C) Applied Probability Trust 2013

\title{
ASYMPTOTIC BEHAVIOUR OF GOSSIP PROCESSES AND SMALL-WORLD NETWORKS
}

\author{
A. D. BARBOUR, ${ }^{*}$ Universität Zürich and National University of Singapore \\ G. REINERT, ${ }^{* *}$ University of Oxford
}

\begin{abstract}
Both small-world models of random networks with occasional long-range connections and gossip processes with occasional long-range transmission of information have similar characteristic behaviour. The long-range elements appreciably reduce the effective distances, measured in space or in time, between pairs of typical points. In this paper we show that their common behaviour can be interpreted as a product of the locally branching nature of the models. In particular, it is shown that both typical distances between points and the proportion of space that can be reached within a given distance or time can be approximated by formulae involving the limit random variable of the branching process.
\end{abstract}

Keywords: Small-world graph; gossip process; branching process approximation

2010 Mathematics Subject Classification: Primary 92H30

Secondary 60K35; 60J85

\section{Introduction}

Moore and Newman [8] introduced a continuous analogue of the Watts and Strogatz [9] 'small-world' model. In this model, a random number of chords are superimposed as shortcuts on a circle $C$ of circumference $L$. The chords have endpoints uniformly and independently distributed on $C$, and the number of chords follows a Poisson distribution $\operatorname{Po}(L \rho / 2)$ with mean $L \rho / 2$ for some $\rho=\rho(L)$. Distance is measured as usual along the circumference, but chords are deemed to be of length 0 , and interest centres on finding the statistics of shortest path distances between pairs of points. A closely related model, the 'great circle model', was introduced somewhat earlier by Ball et al. [2], in the context of epidemics; here the distance between points translates into the time taken for one infected person to infect another. In Barbour and Reinert [4], assuming the expected number $L \rho / 2$ of shortcuts to be large, we proved a distributional approximation for the distance between a randomly chosen pair of points $P$ and $P^{\prime}$, and gave a bound on the order of the error, in terms of the total variation distance. We also showed that analogous results could be proved in higher dimensions by much the same method, when the circle is replaced by a sphere or a torus. It turns out that the reduction in the typical distances between pairs of points that results from introducing shortcuts is still substantial, but less dramatic than in one dimension.

More recently, Chatterjee and Durrett [6] studied a model for the spread of gossip that is the continuous analogue of one of a number of models discussed in Aldous [1]. Here information

Received 23 February 2012; revision received 6 March 2013.

* Postal address: Angewandte Mathematik, Universität Zürich, Winterthurertrasse 190, CH-8057 Zürich, Switzerland. ADB was Saw Swee Hock Professor at the National University of Singapore while part of this work was carried out. Work supported in part by the Australian Research Council grants DP120102728 and DP120102398.

** Postal address: Department of Statistics, University of Oxford, 1 South Parks Road, Oxford OX1 3TG, UK.

Supported in part by the EPSRC and BBSRC through OCISB. 
spreads locally from an individual to their neighbours on the two-dimensional torus, and also occasionally to other, randomly chosen members of the community. Thus, a disc of informed individuals, centred on an initial informant, grows steadily in the torus; long-range transmissions of information occur in a Poisson process, whose rate is proportional to the area (number) of informed individuals, and any such transmission contacts a randomly chosen point of the torus, initiating a new disc of informed individuals. The distinction between this model and the corresponding two-dimensional model in [4] is that, in the 'gossip' model, the Poisson process runs at a rate proportional to the area of the currently informed region; in [4], where the Poisson number of shortcuts is considered to be fixed in advance, the Poisson process corresponds to a process of discovery of shortcuts, and its rate is thus proportional to the length of the boundary of the informed region.

Here we consider the development of such a process $Y$ on a smooth, closed, homogeneous Riemannian manifold $C$ of dimension $d$, such as a sphere or a torus, having large finite volume $|C|=: L$ with respect to its intrinsic metric. We assume that, around each point $P$ of $C$, there is a collection of closed subsets $\mathcal{K}(P, s), s \geq 0$, that are balls of radius $s$ with respect to a metric $d_{C}$ that makes $C$ a geodesic space, and with (intrinsic) volumes $v_{s}(\mathcal{K}):=|\mathcal{K}(P, s)| \sim s^{d} v(\mathcal{K})$ as $s \rightarrow 0$ for some $v(\mathcal{K})>0 ; s$ is thought of as time, and $\{v(\mathcal{K})\}^{1 / d}$ as a (linear) speed of propagation. The metric $d_{C}$ need not be the same as the intrinsic metric; for instance, on the torus, we could consider rectangular as well as circular neighbourhoods. The set $\mathcal{K}(P, s)$ denotes the set of points 'locally' contacted after time $s$ has elapsed following an initial 'long-range' contact at $P$, thought of as 'islands' in $C$, and the complete set of contacts $Y_{P_{0}}(t)$ at time $t$ is the union of these sets growing from an initial point $P_{0}$, and from all long-range contacts made before $t$; we denote its volume by $V_{P_{0}}(t)$. The rate at which long-range contacts are made is proportional either to the area of the boundary of $Y_{P_{0}}(t)$ (small-world processes) or to its volume (gossip processes), and we denote the constant of proportionality by $\rho$; long-range contacts are made with independently and uniformly chosen points of $C$.

The main result of this paper is Theorem 3.2, which establishes a pathwise law of large numbers, together with a rough error bound, for the time evolution of the covered fraction $L^{-1} V_{P_{0}}(t)$ of $C$, in the setting of a quite general gossip process. An analogous result is stated for small-world processes in Theorem 3.3. Theorem 3.2 shows that there exists a random variable $U$ such that, for positive constants $a_{1}, a_{2}$ and $c<\infty$,

$$
\mathbb{P}\left[\sup _{x}\left|L^{-1} V_{P_{0}}\left(\lambda_{0}^{-1}\{\log \Lambda+x\}\right)-h_{d}\left(x+\log C_{d}+U\right)\right|>4 \Lambda^{-a_{1}}\right] \leq c \Lambda^{-a_{2}} .
$$

Here $C_{d}=(d+1)^{-1} d$ !, the function $h_{d}$ depends only on the dimension $d, \lambda_{0}$ is the initial exponential growth rate of the process $Y_{P_{0}}$, and $\Lambda:=L \lambda_{0}^{d} / v(\mathcal{K})$. The value taken by the random variable $U$ is essentially determined by the very early evolution of $Y_{P_{0}}$, and can be thought of as a random delay, caused by early fluctuations in the growth of the process, before the deterministic evolution governed by $h_{d}$ sets in. The function $h_{d}$ is defined through a Laplace transform and satisfies an integral equation, (2.23). Both $U$ and $h_{d}$ have interpretations in terms of an associated Markov branching process $X^{*}$. In the particular case of the torus, our result extends the limit law proved by Chatterjee and Durrett [6], by providing an estimate of the approximation error that is uniform for all time.

Our argument is developed from that in [4]. The key observation is that, at least for a while, the process $Y_{P_{0}}$ can be closely approximated using a Markovian growth and branching process $X^{*}=X_{P_{0}}^{*}$, and that this approximation is accurate enough for the calculations that need 
to be made. The state $X^{*}(t)$ of the Markov branching process at time $t$ consists of $\mathcal{K}\left(P_{0}, t\right)$, together with a collection of some number $n(t)$ of sets of the form $\mathcal{K}\left(P_{j}^{*}, t-\tau_{j}^{*}\right)$, where $0 \leq \tau_{j}^{*} \leq t$ and $P_{j}^{*} \in C$ for each $1 \leq j \leq n(t)$. The $P_{j}^{*}$ are independently and uniformly chosen points of $C$, and arise as the points $\tau_{j}^{*}$ of a Poisson process, whose rate depends on the current state of $X^{*}$. In gossip models, new contacts are made at a rate proportional to the current volume, which, for the process $X^{*}$, is given by

$$
V^{*}(t):=\sum_{j=0}^{n(t)} v_{t-\tau_{j}^{*}}(\mathcal{K}) \sim \sum_{j=0}^{n(t)}\left(t-\tau_{j}^{*}\right)^{d} v(\mathcal{K}) ;
$$

in small-world models, the rate is proportional to the derivative of the volume. The set $Y_{P_{0}}(t)$ can initially be approximated by the union

$$
Y_{P_{0}}^{*}(t):=\bigcup_{j=0}^{n(t)} \mathcal{K}\left(P_{j}^{*}, t-\tau_{j}^{*}\right),
$$

where $\tau_{0}^{*}:=0$ and $P_{0}^{*}=P_{0}$. Indeed, one can take $Y_{P_{0}}(t)=Y_{P_{0}}^{*}(t)$ until the (random) time $\hat{T}$ at which the union in (1.1) ceases to be disjoint. Thereafter, the rate of contacts is smaller in $Y_{P_{0}}$ than it is in $Y_{P_{0}}^{*}$, and the two processes progressively separate.

In [4], the distribution of interpoint distances in the small-world model is determined by running two such branching processes from randomly chosen initial points $P_{0}^{\prime}$ and $P_{0}^{\prime \prime}$, each for a length of time $t^{*}$ at which the mean number of overlaps in (1.1) is of order $O(1)$. At this time, conditionally on the contact times in the two branching processes, the number of permissible overlaps between the sets $Y_{P_{0}^{\prime}}^{*}$ and $Y_{P_{0}^{\prime \prime}}^{*}$-cases in which an island in one branching process is contained within an island in the other could not have arisen in the actual smallworld process - has an approximately Poisson distribution, and the distance between the initial points is greater than $2 t^{*}$ if there are no permissible overlaps. In this way, and by varying the choice of $t^{*}$ appropriately, the distribution of interpoint distances can be approximated, without ever having to go into the dependence structure that becomes important in the process $Y$ at times significantly larger than $t^{*}$. An analogous approach has recently been used in [5], when proving results for the shortest-weight path between two vertices in first passage percolation on a random graph. In contrast, Chatterjee and Durrett [6] go beyond the branching phase in the analysis of $Y$ in their two-dimensional gossip model, and are able to prove a conditional law of large numbers for the fraction of the torus contained in $Y$, given the outcome of the branching phase. They also established the asymptotics of the first time at which $Y$ covers the whole torus.

The point of departure for our argument is that $\mathbb{E}\left\{V_{P_{0}}(t) / L \mid \mathcal{F}_{S}\right\}$, the conditional expectation of the covered fraction at $t$, given the history of $Y_{P_{0}}$ up to time $s$, including the initial point $P_{0}$, is given by

$$
\mathbb{E}\left\{\frac{V_{P_{0}}(t)}{L} \mid \mathcal{F}_{s}\right\}=\mathbb{P}\left[Q \in Y_{P_{0}}(t) \mid \mathcal{F}_{s}\right], \quad t>s,
$$

where $Q$ denotes an independently and uniformly distributed point of $C$. Now, for $t$ in the relevant range (corresponding to $2 t^{*}$ above), we have

$$
Q \in Y_{P_{0}}(t) \text { if and only if } Y_{P_{0}}\left(\frac{1}{2} t\right) \cap \bar{Y}_{Q}\left(\frac{1}{2} t\right) \neq \varnothing,
$$

where $\bar{Y}_{Q}$ is an independent gossip process started from $Q$. The probability of the latter event can then be closely enough approximated by computing the probability of intersection of independent branching processes $X_{P_{0}}^{*}$ and $X_{Q}^{*}$ at time $t / 2$. 
The law of large numbers is proved by using an argument of much the same flavour, since

$$
\mathbb{E}\left\{\left[\frac{V_{P_{0}}(t)}{L}\right]^{2} \mid \mathcal{F}_{s}\right\}=\mathbb{P}\left[\left\{Q \in Y_{P_{0}}(t)\right\} \cap\left\{Q^{\prime} \in Y_{P_{0}}(t)\right\} \mid \mathcal{F}_{s}\right]
$$

for two independent and uniformly distributed points $Q, Q^{\prime} \in C$. Using (1.2) to rewrite this probability, showing that the processes $\bar{Y}_{Q}$ and $\bar{Y}_{Q^{\prime}}$ can be taken to be nearly independent, and using the fact that, for sufficiently large $s$,

$$
\mathbb{P}\left[Y_{P_{0}}\left(\frac{1}{2} t\right) \cap \bar{Y}_{Q}\left(\frac{1}{2} t\right) \neq \varnothing \mid \mathcal{F}_{s}\right] \approx \mathbb{P}\left[Y_{P_{0}}\left(\frac{1}{2} t\right) \cap \bar{Y}_{Q}\left(\frac{1}{2} t\right) \neq \varnothing \mid \mathcal{F}_{t / 2}\right],
$$

it can be shown that

$$
\mathbb{E}\left\{\left[\frac{V_{P_{0}}(t)}{L}\right]^{2} \mid \mathcal{F}_{s}\right\} \approx\left[\mathbb{E}\left\{\left[\frac{V_{P_{0}}(t)}{L}\right] \mid \mathcal{F}_{s}\right\}\right]^{2}
$$

this approximation is made precise in (3.14) and (3.15) of Theorem 3.1. Hence, the conditional variance of $V_{P_{0}}(t) / L$, given the information in $\mathcal{F}_{s}$, is small, and, thus, the value of $V_{P_{0}}(t) / L$ is (almost) fixed. An analogous argument is used, for instance, in Ball et al. [3], where they showed that, in an epidemic in a population of large size $N$, the proportion of individuals ever infected is close either to 0 or to a nonrandom value in $(0,1)$. A by-product of our argument is to identify the solution $h$ of a particular integral equation, which appears in Aldous [1] and also plays a substantial part in the formula given by Chatterjee and Durrett [6], in terms of the Laplace transform of the branching process limit random variable $W$; this function $h$ is just a time translation of $h_{2}$.

The paper is organized as follows. The necessary properties of the branching processes that approximate the early stages of the gossip and small-world processes are established in Section 2. The law of large numbers is then proved in Sections 3.1-3.3. The time until $C$ is completely covered is investigated in Section 3.4, and the paper concludes in Section 3.5 by extending the results to finite subsets of homogeneous manifolds, such as rectangles in $\mathbb{R}^{2}$.

\section{The branching phase}

As in [4], we base our analysis of the coverage process on the pure growth Markov branching process $X^{*}$, which has neighbourhoods with centres independently and uniformly positioned in $C$. In this section, to describe the behaviour of such processes, we specialize to the case of 'flat' manifolds, such as tori, in which

$$
v_{s}(\mathcal{K})=s^{d} v(\mathcal{K}), \quad s \geq 0 .
$$

We later show that this condition can be relaxed substantially, by bounding the branching processes for more general manifolds between processes satisfying condition (2.1) that are close enough for our purposes.

We begin by defining $M_{0}(t):=1+\max \left\{j \geq 0: \tau_{j}^{*} \leq t\right\}$ to be the number of islands in the branching process up to time $t$, and

$$
M_{l}(t)=\sum_{j=1}^{M_{0}(t)}\left(t-\tau_{j-1}^{*}\right)^{l}
$$


to be the sum of the $l$ th powers of their 'radii'. The evolution of the process is then governed by the differential equations

$$
\frac{\mathrm{d}}{\mathrm{d} t} M_{1}(t)=M_{0}(t) \quad \text { for almost every } t, \quad \frac{\mathrm{d}}{\mathrm{d} t} M_{i}(t)=i M_{i-1}(t), \quad i \geq 2,
$$

together with a specification of $M_{0}$. Letting $Z$ denote a unit-rate Poisson process, a small-world process is obtained by setting

$$
\begin{aligned}
M_{0}(t) & =M_{0}(0)+Z\left(\rho v(\mathcal{K}) d \int_{0}^{t} M_{d-1}(u) \mathrm{d} u\right) \\
& =M_{0}(0)+Z\left(\rho v(\mathcal{K})\left[M_{d}(t)-M_{d}(0)\right]\right) ;
\end{aligned}
$$

for a gossip process, we set

$$
\begin{aligned}
M_{0}(t) & =M_{0}(0)+Z\left(\rho v(\mathcal{K}) \int_{0}^{t} M_{d}(u) \mathrm{d} u\right) \\
& =M_{0}(0)+Z\left(\rho(d+1)^{-1} v(\mathcal{K})\left[M_{d+1}(t)-M_{d+1}(0)\right]\right) .
\end{aligned}
$$

In either case, the intensity $\rho$ may depend on $L$.

Equations (2.3)-(2.5) can be rewritten in clearer form by defining $H_{i}(t):=M_{i}(t) \lambda^{i} / i$ ! for $\lambda$ to be suitably chosen, in which case (2.3) reduces to

$$
\frac{\mathrm{d}}{\mathrm{d} t} H_{1}(t)=\lambda H_{0}(t) \quad \text { for almost every } t, \quad \frac{\mathrm{d}}{\mathrm{d} t} H_{i}(t)=\lambda H_{i-1}(t), \quad i \geq 2 ;
$$

for the small-world process, we have

$$
H_{0}(t)=M_{0}(0)+Z\left(d ! \rho v(\mathcal{K}) \lambda^{-d}\left[H_{d}(t)-H_{d}(0)\right]\right)=M_{0}(0)+Z\left(H_{d}(t)-H_{d}(0)\right)
$$

if $\lambda=\lambda_{0}:=(d ! \rho v(\mathcal{K}))^{1 / d}$, and, for the gossip process, we have

$$
\begin{aligned}
H_{0}(t) & =M_{0}(0)+Z\left(d ! \rho v(\mathcal{K}) \lambda^{-d-1}\left[H_{d+1}(t)-H_{d+1}(0)\right]\right) \\
& =M_{0}(0)+Z\left(H_{d+1}(t)-H_{d+1}(0)\right)
\end{aligned}
$$

if $\lambda=\lambda_{0}:=(d ! \rho v(\mathcal{K}))^{1 /(d+1)}$. Note that, since $\rho$ may depend on $L$, so may $\lambda_{0}$.

Remark 2.1. The time-scaled process $\tilde{H}(u):=H(u / \lambda)$ actually satisfies

$$
\frac{\mathrm{d}}{\mathrm{d} t} \tilde{H}_{i}(t)=\tilde{H}_{i-1}(t), \quad i \geq 1, \quad \tilde{H}_{0}(t)=M_{0}(0)+Z\left(\tilde{H}_{r(d)}(t)-\tilde{H}_{r(d)}(0)\right),
$$

where $r(d)=d$ for the small-world process and $d+1$ for the gossip process. Thus, apart from a time change, the processes are the same for all $\lambda$. Despite this, we retain $\lambda_{0}$ in the subsequent discussion, in order to emphasize the connection with the original process.

In either case, the equations for $H=\left(H_{1}, H_{2}, \ldots, H_{r}\right)^{\top}$ are of the form

$$
\frac{\mathrm{d} H}{\mathrm{~d} t}=\lambda_{0} C_{r}\left(H+\hat{h} \varepsilon^{r}\right)=\lambda_{0} C_{r}\left[I+\left(\frac{\hat{h}}{H_{r}}\right) \varepsilon^{r}\left(\varepsilon^{r}\right)^{\top}\right] H,
$$

where $r=r(d), \varepsilon^{i}$ denotes the $i$ th coordinate vector, $C_{r}$ is the $r$-dimensional cyclic permutation matrix, satisfying $C_{r} \varepsilon^{i}=\varepsilon^{i+1}, 1 \leq i \leq r-1$, and $C_{r} \varepsilon^{r}=\varepsilon^{1}$, and

$$
\hat{h}(t):=H_{0}(t)-H_{r}(t)=Z\left(H_{r}(t)-H_{r}(0)\right)-H_{r}(t)+M_{0}(0) .
$$


Without the perturbation $\hat{h}, H$ would have asymptotically exponential growth at rate $\lambda_{0}$, and the ratios of its components would all tend to unity, since the dominant eigenvalue 1 of $C_{r}$ corresponds to the right eigenvector $\mathbf{1}$. For the arguments to come, it will be important to show that, with high enough probability, the asymptotic effect of $\hat{h}$ is just to multiply $H$ by some random constant-a branching random variable $W$-which is not too big. Unless otherwise specified, we henceforth take $M_{0}(0)=1$ and $M_{l}(0)=0$ for all $l \geq 1$, so that we start with just one point $P_{0}$ at $t=0$.

\subsection{Growth bounds for the branching process}

Using the maximum norm $\|\cdot\|$ for $r(d)$ vectors, it follows immediately from (2.9) that

$$
\frac{\mathrm{d}}{\mathrm{d} t}\|H(t)\| \leq \lambda_{0} u(t)\|H(t)\|,
$$

with $u(t):=\left\{1+\left(\hat{h}(t) / H_{r(d)}(t)\right)_{+}\right\}$, so that, by a Gronwall argument,

$$
\|H(t)\| \leq\left\|H\left(t_{0}\right)\right\| \exp \left\{\lambda_{0} \int_{t_{0}}^{t} u(v) \mathrm{d} v\right\}
$$

for any $0 \leq t_{0} \leq t$. Thus, in order to bound the growth of $H$, we shall need to control the quantity $\hat{h}(t) / H_{r(d)}(t)$, which is itself a function of the Poisson process $Z$. To this end, we begin with the following lemma, which controls the extreme fluctuations of $Z$.

Lemma 2.1. Let $Z$ be a unit-rate Poisson process. Then we have the following bounds, uniformly in $t \geq 1$ :

(a) $\mathbb{P}\left[\sup _{u \geq t} u^{-1} Z(u) \geq 2\right] \leq c_{1} \mathrm{e}^{-t / 14}$,

(b) $\mathbb{P}\left[\sup _{u \geq t} u^{1 / 3}\left|u^{-1} Z(u)-1\right| \geq 4\right] \leq c_{2} \mathrm{e}^{-t^{1 / 3} / 5}$,

(c) $\mathbb{P}\left[\inf _{u \geq t} u^{-1} Z(u) \leq \frac{1}{2}\right] \leq c_{3} \mathrm{e}^{-t / 44}$.

Furthermore, for any $U \geq 1$ and $0<\eta \leq \frac{1}{3}$ such that $U\left(2^{\eta}-1\right) \geq 42 \log 2$,

(d) $\mathbb{P}\left[\sup _{u \geq 0}(u \vee 1)^{-(1+\eta) / 2}|Z(u)-u| \geq U\right] \leq c_{4} \mathrm{e}^{-U / 28}$

for a constant $c_{4}$.

Proof. For any $t, \varepsilon>0$, set $u_{j}:=t(1+\varepsilon)^{j}, j \geq 0$. Then it is immediate that

$$
\sup _{u_{j} \leq u \leq u_{j+1}} u^{-1} Z(u) \leq \frac{Z\left(u_{j+1}\right)}{u_{j}} .
$$

Hence, and by the Chernoff inequalities (see [7, Theorem 2.3]),

$$
\begin{aligned}
\mathbb{P}\left[\sup _{u_{j} \leq u \leq u_{j+1}} u^{-1} Z(u) \geq 1+2 \varepsilon\right] & \leq \mathbb{P}\left[u_{j}^{-1} Z\left(u_{j}(1+\varepsilon)\right) \geq 1+2 \varepsilon\right] \\
& \leq \exp \left\{-\frac{\varepsilon^{2} u_{j}}{2+3 \varepsilon}\right\} \\
& =\exp \left\{-\frac{\varepsilon^{2} u_{j-1}}{2+3 \varepsilon}\right\} \exp \left\{-\frac{\varepsilon^{3} u_{j-1}}{2+3 \varepsilon}\right\}
\end{aligned}
$$


and

$$
\begin{aligned}
\mathbb{P}\left[\inf _{u_{j} \leq u \leq u_{j+1}} u^{-1} Z(u) \leq 1-2 \varepsilon\right] & \leq \mathbb{P}\left[\left\{(1+\varepsilon) u_{j}\right\}^{-1} Z\left(u_{j}\right) \leq 1-2 \varepsilon\right] \\
& \leq \exp \left\{-\frac{1}{2} \varepsilon^{2} u_{j}\right\} \\
& =\exp \left\{-\frac{1}{2} \varepsilon^{2} u_{j-1}\right\} \exp \left\{-\frac{1}{2} \varepsilon^{3} u_{j-1}\right\}
\end{aligned}
$$

Adding over $j \geq 0$, the sum is dominated by a geometric progression with common ratio $\exp \left\{-\varepsilon^{3} t /(2+3 \varepsilon)\right\}$, and so it follows that

$$
\mathbb{P}\left[\sup _{u \geq t}\left|u^{-1} Z(u)-1\right| \geq 2 \varepsilon\right] \leq C(\varepsilon, t) \exp \left\{-\frac{\varepsilon^{2} t}{2+3 \varepsilon}\right\},
$$

with $C(\varepsilon, t):=2 /\left(1-\exp \left\{-\varepsilon^{3} t /(2+3 \varepsilon)\right\}\right)$. Taking $\varepsilon=\frac{1}{2}$ gives the first inequality, with $c_{1}:=C\left(\frac{1}{2}, 1\right)$; taking $\varepsilon=\frac{1}{4}$ gives the third inequality, with $c_{3}=C\left(\frac{1}{4}, 1\right)$. For the second inequality, with $t \geq 1, \varepsilon=t^{-1 / 3}$ gives, in particular,

$$
\mathbb{P}\left[\sup _{t \leq u \leq 8 t} u^{1 / 3}\left|u^{-1} Z(u)-1\right| \geq 4\right] \leq C(1,1) \exp \left\{-\frac{1}{5} t^{1 / 3}\right\}
$$

and, thus,

$$
\mathbb{P}\left[\sup _{u \geq t} u^{1 / 3}\left|u^{-1} Z(u)-1\right| \geq 4\right] \leq C(1,1) \sum_{j \geq 0} \exp \left\{-\frac{2^{j} t^{1 / 3}}{5}\right\} \leq c_{2} \exp \left\{-\frac{1}{5} t^{1 / 3}\right\}
$$

with $c_{2}:=C(1,1) /\left(1-\mathrm{e}^{-1 / 5}\right)$, since the ratio of successive terms in $(2.13)$ is at most $\mathrm{e}^{-1 / 5}$.

The fourth inequality is a little trickier. Taking $t \geq 1$ and $\varepsilon=\varepsilon_{t}=U /\left\{2(2 t)^{(1-\eta) / 2}\right\}$ in (2.12), we have

$$
\begin{aligned}
\mathbb{P}\left[\sup _{t \leq u \leq 2 t} u^{(1-\eta) / 2}\left|u^{-1} Z(u)-1\right| \geq U\right] & \leq C\left(\varepsilon_{t}, t\right) \exp \left\{-\frac{2^{-3+\eta} U^{2} t^{\eta}}{2+3 U / 2}\right\} \\
& \leq C\left(\varepsilon_{t}, t\right) \exp \left\{-\frac{1}{28} U t^{\eta}\right\},
\end{aligned}
$$

since, for $U \geq 1, U /(2+3 U / 2) \geq \frac{2}{7}$. For this choice of $\varepsilon, \varepsilon^{2} t$ increases with $t$, but $\varepsilon^{3} t$ decreases; however, since $1-\mathrm{e}^{-x} \geq\left(1-\mathrm{e}^{-1}\right) \min \{1, x\}$ in $x \geq 0$, we have

$$
C(\varepsilon, t) \leq \frac{2}{1-\mathrm{e}^{-1}} \max \left\{1, \frac{2+3 \varepsilon}{\varepsilon^{3} t}\right\} \leq C^{\prime}(\varepsilon, t):=\frac{2}{1-\mathrm{e}^{-1}} \max \left\{1, \frac{8}{\varepsilon^{3} t}\right\},
$$

uniformly in $t \geq 1$; the final inequality is immediate if $\varepsilon \leq 2$, and, for $\varepsilon>2,2+3 \varepsilon<\varepsilon^{3}$. Set $q(t):=C^{\prime}\left(\varepsilon_{t}, t\right) \exp \left\{-U t^{\eta} / 28\right\}$. Then, in the sum $\sum_{j \geq 0} q\left(2^{j}\right)$, the ratios of successive terms are at most

$$
2^{(1-3 \eta) / 2} \exp \left\{-\frac{1}{28} U\left(2^{\eta}-1\right)\right\} \leq \sqrt{2} \exp \left\{-\frac{1}{28} U\left(2^{\eta}-1\right)\right\} \leq \frac{1}{2},
$$

by assumption, so that

$$
\sum_{j \geq 0} q\left(2^{j}\right) \leq 2 q(1) \leq 2^{11 / 2} \frac{16}{1-\mathrm{e}^{-1}} \exp \left\{-\frac{U}{28}\right\} .
$$

Since, by an exponential moment inequality, $\mathbb{P}[Z(1)>U] \leq c \mathrm{e}^{-U / 14}$ with $c:=\mathrm{e}^{x-1}$ and $x=\mathrm{e}^{1 / 14}$, the proof of the fourth inequality is complete. 
Based on Lemma 2.1, we can now prove growth bounds for the Markov branching process. Here we allow for quite general initial conditions. For ease of reference, for any $K \geq 1$ and $0<\eta<1$, we define the events

$$
\begin{aligned}
A_{K, s}^{(1)} & :=\left\{\mathrm{e}^{-\lambda_{0} s}\|H(s)\| \leq K\right\}, \quad A_{K, s}^{(2)}:=\left\{H_{r(d)}(s) \geq K\right\}, \\
A_{K, \eta, s}^{(3)} & :=\left\{\sup _{0 \leq u \leq H_{r(d)}(s)}(u \vee 1)^{-(1+\eta) / 2}|Z(u)-u| \leq K^{(1-\eta) / 2}\right\}, \\
A^{\prime}(K, s) & :=\left\{\exp \left\{-\lambda_{0}(t-s)\left(1+\varepsilon_{K}\right)\right\}\|H(t)\| \leq\|H(s)\| \text { for all } t>s\right\},
\end{aligned}
$$

where $\varepsilon_{K}:=5 K^{-1 / 3}$, and we write

$$
A_{K, s}:=A_{\theta K, s}^{(1)} \cap A_{K, s}^{(2)} \cap A_{K, \varepsilon_{K}, s}^{(3)} \in \mathcal{F}_{s},
$$

where $\mathcal{F}_{s}$ denotes the history of $X^{*}$ up to time $s$, and $\theta:=C_{a} \mathrm{e}^{1 / 80}$, with $C_{a}$ as defined below.

Theorem 2.1. For any $K \geq 1$ and any $0 \leq s<t$, we have

(a) $\mathbb{P}\left[\exp \left\{-\lambda_{0}(t-s)\left(1+\varepsilon_{K}\right)\right\}\|H(t)\| \leq \max \left\{C_{a} K,\|H(s)\|\right\}\right.$ for all $\left.t>s\right] \geq 1-c_{a} \mathrm{e}^{-K^{1 / 3} / 5}$ for suitable constants $C_{a}:=3 \exp \left\{(r(d) !)^{1 / r(d)}\right\}$ and $c_{a}$. Furthermore,

(b) $\mathbb{P}\left[A^{\prime}(K, s) \mid \mathcal{F}_{S} \cap A_{K, s}^{(2)}\right] \geq 1-c_{2} \mathrm{e}^{-K^{1 / 3} / 5}$.

Proof. For $K \geq 1$, define $\tau_{K, s}:=\inf \left\{t \geq s: H_{r(d)}(t) \geq K\right\}$, and suppose first that $\tau_{K, s}>s$. Then $H_{r(d)}(t) \leq K$ if $s \leq t \leq \tau_{K, s}$, and, thus, for all such $t$,

$$
H_{0}(t) \leq 1+Z(K) \leq 3 K,
$$

by Lemma 2.1(a), on a set $A_{1}(K)$ of probability at least $1-c_{1} \exp \{-K / 14\}$. Moreover, from the definition of $H_{i}(t)$ and by Hölder's inequality applied to $M_{i}(t)$, we have

$$
H_{i}(t) \leq \frac{(r(d) !)^{i / r(d)}}{i !} H_{r(d)}(t)^{i / r(d)} H_{0}(t)^{1-i / r(d)}, \quad 1 \leq i<r(d) .
$$

Hence, on $A_{1}(K)$, it follows for all $s \leq t \leq \tau_{K, s}$ that

$$
\|H(t)\| \leq \max _{1 \leq i \leq r(d)}\left\{\frac{\left\{(r(d) !)^{1 / r(d)}\right\}^{i}}{i !} K^{i / r(d)}(3 K)^{1-i / r(d)}\right\} \leq 3 \exp \left\{(r(d) !)^{1 / r(d)}\right\} K .
$$

Now, from Lemma 2.1(b), it follows that

$$
\begin{aligned}
u(t) & =1+\left(\frac{\hat{h}(t)}{H_{r(d)}(t)}\right)_{+} \\
& \leq \max \left\{1, K^{-1}+\frac{Z\left(H_{r(d)}(t)\right)}{H_{r(d)}(t)}\right\} \\
& \leq 1+5 K^{-1 / 3} \\
& =1+\varepsilon_{K}
\end{aligned}
$$

for all $t \geq \tau_{K, s}$, on an event $A_{2}(K)$ of probability at least $1-c_{2} \mathrm{e}^{-K^{1 / 3} / 5}$. By (2.11), this implies that, on $A_{2}(K) \cap A_{1}(K)$, for all $t \geq \tau_{K, s}$,

$$
\|H(t)\| \leq\left\|H\left(\tau_{K, s}\right)\right\| \exp \left\{\lambda_{0}(t-s)\left(1+\varepsilon_{K}\right)\right\} .
$$


If $\tau_{K, s}>s$, by (2.17), this in turn implies that, on $A_{2}(K) \cap A_{1}(K)$,

$$
\|H(t)\| \leq 3 \exp \left\{(r(d) !)^{1 / r(d)}\right\} K \exp \left\{\lambda_{0}(t-s)\left(1+\varepsilon_{K}\right)\right\}
$$

for all $t \geq s$; if $\tau_{K, s}=s$, we simply have $\|H(t)\| \leq\|H(s)\| \exp \left\{\lambda_{0}(t-s)\left(1+\varepsilon_{K}\right)\right\}$. This establishes part (a).

For part (b), if $H_{r(d)}(s) \geq K$, it follows as above that

$$
\mathbb{P}\left[u(t) \leq 1+\varepsilon_{K} \text { for all } t \geq s \mid \mathcal{F}_{S}\right] \geq 1-c_{2} \mathrm{e}^{-K^{1 / 3} / 5},
$$

and, if this is the case then

$$
\exp \left\{-\lambda_{0}(t-s)\left(1+\varepsilon_{K}\right)\right\}\|H(t)\| \leq\|H(s)\| \quad \text { for all } t>s
$$

follows from (2.11). This completes the proof.

Theorem 2.1 translates into bounds on the values of $\hat{h}(t)$, defined in (2.10).

Corollary 2.1. Given any $\varepsilon>0$, there exists a random variable $H^{\varepsilon}$ such that

(a) $|\hat{h}(t)| \leq H^{\varepsilon} \exp \left\{\frac{1}{2} \lambda_{0}(1+\varepsilon) t\right\}$ a.s. for all $t>0$.

In addition, for any $K \geq 1$,

(b) $\mathbb{E}\left\{|\hat{h}(t)| I\left[A^{\prime}(K, s)\right] \mid \mathcal{F}_{s} \cap A_{K, s}\right\} \leq 2\left\{(\theta K)^{1 / 2}+K\right\} \exp \left\{\frac{1}{2} \lambda_{0}\left(1+\varepsilon_{K}\right) t\right\}$.

Proof. Note that, from Theorem 2.1(a), given any $K_{0} \geq 1$, there exists a.s. a (random) $K \geq K_{0}$ such that

$$
\sup _{t>0} \mathrm{e}^{-\lambda_{0} t\left(1+\varepsilon_{K}\right)}\|H(t)\|<\infty
$$

since, for each $K$, the supremum is finite with probability at least $1-c_{a} \mathrm{e}^{-K^{1 / 3} / 5}$. Hence, for any $\varepsilon>0$,

$$
H_{\varepsilon}^{\prime}:=\max \left\{1, \sup _{t>0}\left\{\mathrm{e}^{-\lambda_{0}(1+\varepsilon) t}\|H(t)\|\right\}\right\}<\infty \quad \text { a.s. }
$$

This in turn implies that, given any $\varepsilon>0$,

$$
|\hat{h}(t)-1|=\left|Z\left(H_{r(d)}(t)\right)-H_{r(d)}(t)\right| \leq \sup _{0 \leq u \leq H_{\varepsilon / 2}^{\prime}}{\exp \left\{\lambda_{0}(1+\varepsilon / 2) t\right\}}_{|Z(u)-u|} \mid \text { for all } t>0 .
$$

Now, from the law of the iterated logarithm for the Poisson process, for any $\eta>0$,

$$
H_{\eta}^{\prime \prime}:=\sup _{u \geq 1}\left\{u^{-\eta-1 / 2}|Z(u)-u|\right\}<\infty \quad \text { a.s. }
$$

Part (a) thus follows because

$$
\begin{aligned}
\sup _{t \geq 1}\{ & \left.\exp \left\{-\frac{1}{2} \lambda_{0}(1+\varepsilon) t\right\}|\hat{h}(t)|\right\} \\
& \leq 1+H_{\eta}^{\prime \prime} H_{\varepsilon / 2}^{\prime} \sup _{t \geq 1}\left\{\exp \left\{\left[\left(\frac{1}{2}+\eta\right)\left(1+\frac{1}{2} \varepsilon\right)-\frac{1}{2}(1+\varepsilon)\right] \lambda_{0} t\right\}\right\} \\
& <\infty,
\end{aligned}
$$

if $\eta$ is chosen such that $\eta(1+\varepsilon / 2)<\varepsilon / 4$. 
For part (b), if $\tilde{Z}$ is a Poisson process of rate 1, the Doob-Kolmogorov inequality gives

$$
\mathbb{P}\left[\sup _{0 \leq u \leq U}|\tilde{Z}(u)-u|>x\right] \leq \min \left\{U x^{-2}, 1\right\},
$$

and, hence, $\mathbb{E}\left\{\sup _{0 \leq u \leq U}|\tilde{Z}(u)-u|\right\} \leq 2 \sqrt{U}$. Thus, from (2.15), we have

$$
\begin{aligned}
& \mathbb{E}\left\{|\hat{h}(t)-\hat{h}(s)| I\left[A^{\prime}(K, s)\right] \mid \mathcal{F}_{s} \cap A_{K, s}\right\} \\
& \quad \leq \mathbb{E}\left\{\sup _{0 \leq u \leq\|H(s)\| \exp \left\{\lambda_{0}\left(1+\varepsilon_{K}\right)(t-s)\right\}}|\tilde{Z}(u)-u| \mid \mathcal{F}_{s} \cap A_{K, s}\right\} \\
& \quad \leq 2(\theta K)^{1 / 2} \exp \left\{\frac{1}{2} \lambda_{0}\left(1+\varepsilon_{K}\right) t\right\},
\end{aligned}
$$

where $\tilde{Z}(u):=Z\left(u+H_{r(d)}(s)\right)-Z\left(H_{r(d)}(s)\right)$. Since also, on $A_{K, s}$,

$$
|\hat{h}(s)-1| \leq H_{r(d)}(s)^{\left(1+\varepsilon_{K}\right) / 2} K^{\left(1-\varepsilon_{K}\right) / 2} \leq K \exp \left\{\frac{1}{2} \lambda_{0} s\left(1+\varepsilon_{K}\right)\right\},
$$

the proof is completed.

We now turn to investigating the detail of the long-term behaviour of the vector $\mathrm{e}^{-\lambda_{0} t} H(t)$. To start with, recalling (2.9) and writing $W_{*}(t):=\mathrm{e}^{-\lambda_{0} t} \mathbf{1}^{\top} H(t)$, we have

$$
\frac{\mathrm{d} W_{*}}{\mathrm{~d} t}=\lambda_{0} \mathrm{e}^{-\lambda_{0} t} \hat{h}(t),
$$

and, in view of Corollary 2.1(a), it follows that $W_{*}(\infty):=\lim _{t \rightarrow \infty} W_{*}(t)$ exists and is finite a.s. Note that (2.18) can also be written as

$$
\frac{\mathrm{d} W_{*}}{\mathrm{~d} t}=\lambda_{0} \mathrm{e}^{-\lambda_{0} t}\left\{H_{0}(t)-H_{r(d)}(t)\right\},
$$

from which, by partial integration, it follows that

$$
W_{*}(\infty)=1+\int_{0}^{\infty} \mathrm{e}^{-\lambda_{0} t}\left\{\mathrm{~d} H_{0}(t)-\lambda_{0} H_{r(d)-1}(t) \mathrm{d} t\right\},
$$

identifying $W_{*}(\infty)$ as $r(d) W$, where $W$ is the limiting random variable defined in [4, Theorem 4.1$]$.

In order show how $W_{*}(\infty)$ determines the limiting behaviour of $\mathrm{e}^{-\lambda_{0} t} H(t)$, we first need the following observations. Integrating (2.9), and noting that $H(0)=0$ because $M_{l}(0)=0$ for all $l \geq 1$, we have

$$
\mathrm{e}^{-\lambda_{0} C_{r(d)} t} H(t)=\int_{0}^{t} \lambda_{0} C_{r(d)} \mathrm{e}^{-\lambda_{0} C_{r(d)} u} \varepsilon^{r(d)} \hat{h}(u) \mathrm{d} u,
$$

from which it follows that

$$
\mathrm{e}^{-\lambda_{0} t} H(t)=\mathrm{e}^{-\lambda_{0} t} \int_{0}^{t} \lambda_{0} C_{r(d)} \mathrm{e}^{\lambda_{0} C_{r(d)}(t-u)} \varepsilon^{r(d)} \hat{h}(u) \mathrm{d} u .
$$

Since, from (2.10), $\mathbb{E} \hat{h}(u)=1$ for all $u \geq 0$, it follows directly that

$$
\mathrm{e}^{-\lambda_{0} t}\|\mathbb{E} H(t)\| \leq \mathrm{e}^{-\lambda_{0} t} \int_{0}^{t} \lambda_{0} \mathrm{e}^{\lambda_{0}(t-u)}\left\|\varepsilon^{r(d)}\right\| \mathrm{d} u \leq 1,
$$

so that $\mathrm{e}^{-\lambda_{0} t} H(t)$ is uniformly bounded in expectation. With the help of these facts, we can now establish the following theorem. 
Theorem 2.2. As $t \rightarrow \infty, W(t):=r(d) \mathrm{e}^{-\lambda_{0} t} H(t) \rightarrow W_{*}(\infty) 1$ a.s. Furthermore, for any $0<s<t$,

(a) $\|W(t)-W(s)\|=O\left(\exp \left\{-\frac{1}{2}(1-\varepsilon) \lambda_{0} s\right\}\right)$ a.s.

for any $\varepsilon>0$ if $r(d) \leq 6$, and

(b) $\|W(t)-W(s)\|=O\left(\exp \left\{-(1-\cos (2 \pi / r(d))) \lambda_{0} s\right\}\right)$ a.s.

if $r(d) \geq 7$. Finally, for $A^{\prime}(K, s)$ as defined in (2.15),

(c) $\mathbb{E}\left\{\|W(t)-W(s)\| I\left[A^{\prime}(K, s)\right] \mid \mathcal{F}_{s} \cap A_{K, s}\right\}=O\left(K \exp \left\{-\beta_{r(d)} \lambda_{0} s\right\}\right)$,

where $\beta_{r}=\frac{1}{2}\left(1-\varepsilon_{K}\right)$ if $r \leq 6$, and $\beta_{r}=(1-\cos (2 \pi / r))$ if $r \geq 7$ and $K^{1 / 3} \geq$ $5 /\{2 \cos (2 \pi / 7)-1\}$.

Proof. Since the eigenvalues of $C_{r}$ are the $r$ th roots $\omega_{j}:=\mathrm{e}^{2 \pi(j-1) \mathrm{i} / r}$ of unity, $1 \leq j \leq r$, and the corresponding eigenvectors $e^{(j)}:=r^{-1 / 2}\left(\omega_{j}^{-1}, \omega_{j}^{-2}, \ldots, \omega_{j}^{-(r-1)}, 1\right)$ are (complex) orthonormal, we can write $\mathrm{e}^{-\lambda_{0} t} H(t)=\sum_{j=1}^{r(d)} f_{j}(t) e^{(j)}$ with $f_{j}(t):=\mathrm{e}^{-\lambda_{0} t}\left\{\bar{e}^{(j)}\right\}^{\top} H(t)$ and use (2.19) to compute the $f_{j}(t)$.

Taking $j=1$ first, premultiplying $\mathrm{e}^{-\lambda_{0} t} H(t)$ by $r(d)^{1 / 2}\left\{e^{(1)}\right\}^{\top}=\mathbf{1}^{\top}$ gives

$$
r(d)^{1 / 2} f_{1}(t)=\mathrm{e}^{-\lambda_{0} t} \int_{0}^{t} \lambda_{0} \mathrm{e}^{\lambda_{0}(t-u)} \mathbf{1}^{\top} \varepsilon^{r(d)} \hat{h}(u) \mathrm{d} u=\int_{0}^{t} \lambda_{0} \mathrm{e}^{-\lambda_{0} u} \hat{h}(u) \mathrm{d} u .
$$

This expression converges a.s. to $W_{*}(\infty)$ in view of (2.18) and, hence, $f_{1}(t) \rightarrow r(d)^{-1 / 2} W_{*}(\infty)$ a.s. as $t \rightarrow \infty$. Indeed, we have a little more: for any $s<t$, from Corollary 2.1(a),

$$
r(d)^{1 / 2}\left|f_{1}(t)-f_{1}(s)\right| \leq \int_{s}^{t} \lambda_{0} \mathrm{e}^{-\lambda_{0} u}|\hat{h}(u)| \mathrm{d} u \leq \frac{2 H^{\varepsilon}}{1-\varepsilon} \exp \left\{-\frac{1}{2}(1-\varepsilon) \lambda_{0} s\right\} \quad \text { a.s. }
$$

for any $\varepsilon>0$. For $2 \leq j \leq r(d)$, we have

$$
r(d)^{1 / 2} f_{j}(t)=\mathrm{e}^{-\lambda_{0} t} \int_{0}^{t} \lambda_{0} \omega_{j} \mathrm{e}^{\lambda_{0} \omega_{j}(t-u)} \hat{h}(u) \mathrm{d} u,
$$

giving

$$
r(d)^{1 / 2}\left|f_{j}(t)\right| \leq \mathrm{e}^{-\lambda_{0} t} \int_{0}^{t} \lambda_{0} \mathrm{e}^{\lambda_{0} \rho_{j}(t-u)}|\hat{h}(u)| \mathrm{d} u,
$$

where $\rho_{j}:=\cos \left\{2 \pi(j-1) / r_{(d)}\right\}$.

Corollary 2.1(a) now implies that, a.s.,

$$
\int_{0}^{t} \mathrm{e}^{-\lambda_{0} \rho u}|\hat{h}(u)| \mathrm{d} u= \begin{cases}O(1) & \text { if } \rho>\frac{1}{2}, \\ O\left(\exp \left\{\lambda_{0} t\left(\frac{1}{2}-\rho+\varepsilon\right)\right\}\right) & \text { if } \rho \leq \frac{1}{2} \text { and } \varepsilon>0 .\end{cases}
$$

The estimates given in parts (a) and (b) follow directly, since $\rho_{2}>\frac{1}{2}$ if $r(d) \geq 7$, and

$$
\lim _{t \rightarrow \infty} \mathrm{e}^{-\lambda_{0} t} H(t)=r(d)^{-1} W_{*}(\infty) \mathbf{1} \quad \text { a.s. }
$$

is immediate. The bound given in part (c) follows in a similar way, but using Corollary 2.1(b) in place of Corollary 2.1(a); the final condition on $K$ is merely to ensure that $\beta_{7}<\frac{1}{2}\left(1-\varepsilon_{K}\right)$. 
Remark 2.2. Note, in particular, that the distribution of $W_{*}(\infty)$ is the same, for a given value of $r(d)$, irrespective of the value of $\lambda_{0}$, since $W\left(u / \lambda_{0}\right)=r(d) \mathrm{e}^{-u} H\left(u / \lambda_{0}\right)=r(d) \mathrm{e}^{-u} \tilde{H}(u)$, with $\tilde{H}$ as defined in Remark 2.1. We shall denote a random variable with this distribution by $W_{*}^{[r(d)]}(\infty)$, if the dimension needs to be emphasized.

For use in Section 3, we define

$$
\phi(\theta):=\mathbb{E}\left\{\mathrm{e}^{-\theta W_{*}(\infty)} \mid M_{0}(0)=1, M_{j}(0)=0, j \geq 1\right\} .
$$

The function $\phi$, being the Laplace transform of a branching process limit random variable, can, as usual, be expressed as the solution of an implicit equation. This is based on the observation that, because of the branching property,

$$
W_{*}(\infty) \stackrel{\mathrm{D}}{=} \sum_{j \geq 1} \mathrm{e}^{-\lambda_{0} \tau_{j}} W_{*}^{(j)},
$$

where $\left(W_{*}^{(j)}, j \geq 1\right)$ are independent copies of $W_{*}(\infty)$ and $\left(\tau_{j}, j \geq 1\right)$ are the event times in a Poisson process on $\mathbb{R}_{+}$. For the gossip process in dimension $d$, the Poisson process has intensity

$$
\rho v(\mathcal{K}) u^{d}=\frac{\lambda_{0}^{d+1} u^{d}}{d !}, \quad u>0 .
$$

This implies that the Laplace transform $\phi$ satisfies

$$
\begin{aligned}
\phi(\theta) & =\exp \left\{-\int_{0}^{\infty} \frac{1}{d !}\left\{1-\phi\left(\theta \mathrm{e}^{-\lambda_{0} u}\right)\right\} \lambda_{0}^{d+1} u^{d} \mathrm{~d} u\right\} \\
& =\exp \left\{-\int_{0}^{\infty} \frac{1}{d !}\left\{1-\phi\left(\theta \mathrm{e}^{-x}\right)\right\} x^{d} \mathrm{~d} x\right\},
\end{aligned}
$$

with $\left.-\phi^{\prime}(0)=\mathbb{E}\left\{W_{*}(\infty)\right\} \mid M_{0}(0)=1, M_{j}(0)=0, j \geq 1\right\}=1$. If we define $h(t):=$ $1-\phi\left(\mathrm{e}^{t}\right)$ then $h$ satisfies the equation

$$
h(t)=1-\exp \left\{-\int_{0}^{\infty} \frac{x^{d}}{d !} h(t-x) \mathrm{d} x\right\},
$$

with $\lim _{t \rightarrow-\infty} \mathrm{e}^{-t} h^{\prime}(t)=1$; in the case $d=2,(2.23)$ is the equation that appears in Chatterjee and Durrett [6, Lemma 1.1]. Note that the functions $\phi=\phi_{d}$ and $h=h_{d}$ thus depend only on the dimension $d$, and not on the choice of neighbourhoods, and, hence, that this is true also of the distribution of $W_{*}(\infty)$.

It follows from (2.22) that the lower tail of $W_{*}(\infty)$ can be easily bounded:

$$
\begin{aligned}
\mathbb{P}\left[W_{*}(\infty) \leq w\right] & \leq \operatorname{e} \phi\left(\frac{1}{w}\right) \\
& =\operatorname{eexp}\left\{-\int_{0}^{\infty} \frac{1}{d !}\left\{1-\phi\left(\frac{\mathrm{e}^{-x}}{w}\right)\right\} x^{d} \mathrm{~d} x\right\} \\
& \leq \operatorname{eexp}\left\{-\int_{0}^{-\log w} \frac{1}{d !}\{1-\phi(1)\} x^{d} \mathrm{~d} x\right\} \\
& \leq \operatorname{e}\left(\exp \left\{-c\left(\log \left(\frac{1}{w}\right)\right)^{d+1}\right\}\right) \quad \text { for } c:=\frac{1-\phi(1)}{(d+1) !}
\end{aligned}
$$


Thus, $\mathbb{P}\left[W_{*}(\infty) \leq w\right]$ goes to 0 faster than any power of $w$ as $w \rightarrow 0$ for all $d \geq 1$. For the upper tail,

$$
\mathbb{P}\left[W_{*}(\infty) \geq w\right] \leq \frac{1}{w}
$$

is immediate from Markov's inequality.

Our final result of the section is a lower bound on the growth of $H$, needed later to show that $\mathbb{P}\left[A_{K, s}^{(2)}\right]$ is large enough, if $s$ is not too small.

Lemma 2.2. Let $\tau_{K}^{r(d)}$ denote the time taken for $H_{r(d)}$ to first reach a given level $K$. Then there is a constant $c_{c}:=2(r(d) !)^{1 / r(d)} / \log \left(\frac{6}{5}\right)$ such that

$$
\mathbb{P}\left[\tau_{K}^{r(d)} \geq c_{c} \lambda_{0}^{-1} R\right] \leq 2 K \mathrm{e}^{-R}
$$

for all $R \geq 1$.

Proof. Define $t_{0}:=\lambda_{0}^{-1}(r(d) !)^{1 / r(d)}$, so that, starting $X^{*}$ with a single particle at 0 , we have $H_{r(d)}\left(t_{0}\right) \geq 1$ a.s. Then it is immediate that the process $M_{0}$ is stochastically bounded below by a process $\tilde{M}_{0}$, where $\left(\tilde{M}_{0}\left(n t_{0}\right), n \geq 0\right)$ is a Galton-Watson process with $\tilde{M}_{0}(0)=1$ and with offspring distribution $p_{1}=\left(1-\bar{p}_{2}\right)=\mathrm{e}^{-1}$, and that, for $n t_{0} \leq t<(n+1) t_{0}$, $\tilde{M}_{0}(t)=\tilde{M}_{0}\left(n t_{0}\right)$. Furthermore, for all $n \geq 1, H_{r(d)}\left(n t_{0}\right) \geq \tilde{M}_{0}\left((n-1) t_{0}\right)$. Now, since $\operatorname{Bi}\left(m, \frac{1}{2}\right)\{[m / 2, m]\} \geq \frac{1}{2}$ for all $m \geq 1$, a calculation shows that

$$
\mathbb{E}\left\{\frac{1}{\tilde{M}_{0}\left((n+1) t_{0}\right)} \mid \tilde{M}_{0}\left(n t_{0}\right)=m\right\} \leq m^{-1}\left(\frac{1}{2}+\frac{1}{3}\right),
$$

from which it follows that $\left(\left(\frac{6}{5}\right)^{n} / \tilde{M}_{0}\left(n t_{0}\right), n \geq 0\right)$ is a nonnegative supermartingale, with initial value 1 . So, defining $v_{K}:=\min \left\{n: \tilde{M}_{0}\left(n t_{0}\right) \geq K\right\}$, and noting also that $\tilde{M}_{0}\left(v_{K} t_{0}\right) \leq 2 K$, it follows from the optional stopping theorem that

$$
\mathbb{E}\left\{\left(\frac{6}{5}\right)^{v_{K}}\right\} \leq 2 K .
$$

It thus follows that $\mathbb{P}\left[v_{K} \geq R / \log \left(\frac{6}{5}\right)\right] \leq 2 K \mathrm{e}^{-R}$ for any $R>0$. As $\tau_{K}^{r(d)} / t_{0} \leq v_{K}+1$, we have

$$
\mathbb{P}\left[\tau_{K}^{r(d)} \geq t_{0}\left\{1+\frac{R}{\log (6 / 5)}\right\}\right] \leq 2 K \mathrm{e}^{-R}
$$

also. Since $R+\log \left(\frac{6}{5}\right) \leq 2 R$ in $R \geq 1$, the lemma follows.

\subsection{Intersection asymptotics}

The branching process $X^{*}$ gives a useful approximation to $Y$ as long as it has only a few pairs of self-intersecting islands. Thus, our asymptotics for the branching process are of most interest in the time before self-intersections in $X^{*}$ become plentiful. To see when this is, we need to derive formulae for the mean number of pairs of self-intersecting islands of $X^{*}$ at a given time $t$, and the number of islands of one process $X_{1}^{*}$ that intersect islands of another, independent process $X_{2}^{*}$.

To this end, we strengthen the assumption that $v_{s}(\mathcal{K}) \sim s^{d} v(\mathcal{K})$ as $s \rightarrow 0$ by assuming that

$$
\left|\frac{s^{-d} v_{s}(\mathcal{K})}{v(\mathcal{K})}-1\right| \leq c_{g}\left(s\left\{\frac{v(\mathcal{K})}{L}\right\}^{1 / d}\right)^{\gamma_{g}}, \quad s>0,
$$

for some $\gamma_{g}>0$. Two subsets $\mathcal{K}(P, t)$ and $\mathcal{K}(Q, u)$ intersect when $P \in \mathcal{K}(Q, t+u)$, or, equivalently, when $Q \in \mathcal{K}(P, t+u)$, so that the probability of intersection if $P$ and $Q$ are 
chosen independently and uniformly distributed on $C$ (with respect to the intrinsic volume) is given by

$$
q_{L}(t, u):=L^{-1} v_{t+u}(\mathcal{K})=L^{-1} v(\mathcal{K})(t+u)^{d}\left(1+R_{L}(t, u)\right),
$$

where

$$
\left|R_{L}(t, u)\right| \leq c_{g}\left\{(t+u)\left(\frac{v(\mathcal{K})}{L}\right)^{1 / d}\right\}^{\gamma_{g}} .
$$

We begin by supposing that $c_{g}=0$. In this case, the number $N(t)$ of self-intersecting pairs of islands in a branching process at time $t$, conditional on having $M_{0}(t)=n+1$ islands originating at times $\tau_{0}^{*}=0, \tau_{1}^{*}, \ldots, \tau_{n}^{*} \leq t$, has mean given by

$$
\begin{aligned}
\mathbb{E}\{N(t) & \left.\mid M_{0}(t)=n+1, \tau_{1}^{*}, \ldots, \tau_{n}^{*}\right\} \\
= & \mu\left(n+1 ; t, t-\tau_{1}^{*}, \ldots, t-\tau_{n}^{*}\right) \\
:= & \sum_{i=0}^{n-1} \sum_{j=i+1}^{n} L^{-1} v(\mathcal{K}) \sum_{l=0}^{d}\left(\begin{array}{l}
d \\
l
\end{array}\right)\left(t-\tau_{i}^{*}\right)^{l}\left(t-\tau_{j}^{*}\right)^{d-l} \\
= & \frac{1}{2} L^{-1} v(\mathcal{K}) \sum_{l=0}^{d}\left(\begin{array}{l}
d \\
l
\end{array}\right)\left\{M_{l}(t) M_{d-l}(t)-M_{d}(t)\right\} .
\end{aligned}
$$

Similarly, again if $c_{g}=0$, for two independent branching processes, one that has developed to time $t$ and has $M_{0}(t)=m+1$ islands originating at times $0, \tau_{1}^{*}, \ldots, \tau_{m}^{*} \leq t$, and the other that has developed to time $s$ and has $\tilde{M}_{0}(s)=n+1$ islands originating at times $\sigma_{0}^{*}=0, \sigma_{1}^{*}, \ldots, \sigma_{n}^{*} \leq s$, the conditional mean of the number $N(t, s)$ of intersecting pairs is given by

$$
\begin{aligned}
& \mathbb{E}\left\{N(t, s) \mid M_{0}(t)=m+1, \tau_{1}^{*}, \ldots, \tau_{m}^{*}, \tilde{M}_{0}(s)=n+1, \sigma_{1}^{*}, \ldots, \sigma_{n}^{*}\right\} \\
& \quad=\mu^{\prime}\left(m+1, n+1 ; t, t-\tau_{1}^{*}, \ldots, t-\tau_{m}^{*} ; s, s-\sigma_{1}^{*}, \ldots, s-\sigma_{n}^{*}\right) \\
& \quad:=\sum_{i=0}^{m} \sum_{j=0}^{n} L^{-1} v(\mathcal{K}) \sum_{l=0}^{d}\left(\begin{array}{l}
d \\
l
\end{array}\right)\left(t-\tau_{i}^{*}\right)^{l}\left(s-\sigma_{j}^{*}\right)^{d-l} \\
& \quad=L^{-1} v(\mathcal{K}) \sum_{l=0}^{d}\left(\begin{array}{l}
d \\
l
\end{array}\right) M_{l}(t) \tilde{M}_{d-l}(s),
\end{aligned}
$$

where $\tilde{M}_{l}(s):=\sum_{j=0}^{n}\left(s-\sigma_{j}^{*}\right)^{l}$. Thus, the quantities $M_{l}(\cdot)$ of the previous section are exactly the quantities needed for making such computations. If $c_{g}>0$, we instead have

$$
\begin{aligned}
& \left|\mathbb{E}\left\{N(t) \mid M_{0}(t)=n+1, \tau_{1}^{*}, \ldots, \tau_{n}^{*}\right\}-\mu\left(n+1 ; t, t-\tau_{1}^{*}, \ldots, t-\tau_{n}^{*}\right)\right| \\
& \leq\left(\begin{array}{l}
n \\
2
\end{array}\right) c_{g}\left\{\frac{(2 t)^{d} v(\mathcal{K})}{L}\right\}^{1+\gamma_{g} / d}, \\
& \mid \mathbb{E}\left\{N(t, s) \mid M_{0}(t)=m+1, \tau_{1}^{*}, \ldots, \tau_{m}^{*}, \tilde{M}_{0}(s)=n+1, \sigma_{1}^{*}, \ldots, \sigma_{n}^{*}\right\} \\
& -\mu^{\prime}\left(m+1, n+1 ; t, t-\tau_{1}^{*}, \ldots, t-\tau_{m}^{*} ; s, s-\sigma_{1}^{*}, \ldots, s-\sigma_{n}^{*}\right) \mid \\
& \leq m n c_{g}\left\{\frac{(2 t)^{d} v(\mathcal{K})}{L}\right\}^{1+\gamma_{g} / d} .
\end{aligned}
$$


Now, from Theorem 2.2 we have

$$
M_{l}(t) M_{d-l}(t) \sim l !(d-l) ! \lambda_{0}^{-d} r(d)^{-2} \mathrm{e}^{2 \lambda_{0} t}\left\{W_{*}(\infty)\right\}^{2} \quad \text { as } t \rightarrow \infty,
$$

so that the mean number of self-intersections is small up until times $t$ at which $\mathrm{e}^{2 \lambda_{0} t}$ becomes comparable with

$$
\Lambda:=\frac{L \lambda_{0}^{d}}{v(\mathcal{K})},
$$

or, equivalently, until times of the form

$$
t=t_{\Lambda, x}:=\frac{1}{2 \lambda_{0}}\{\log \Lambda+x\}
$$

for $|x|$ thought of as small compared to $\log \Lambda$. As can be seen from (2.9), $\lambda_{0}^{-1}$ is the time scale for the growth of $H(\cdot)$, or, equivalently, in view of (2.6) and (2.7), the time scale on which the first new contact occurs. Correspondingly, $v(\mathcal{K}) \lambda_{0}^{-d}$ represents the scale for the size of the initial island at the time when the first new contact occurs. For the sort of asymptotics to be discussed here, it is natural to require that this size is small when compared to the total size $L$ of $C$, so that many islands are involved in the covering of $C$; hence, we shall think of the ratio $\Lambda=L /\left\{v(\mathcal{K}) \lambda_{0}^{-d}\right\}$ as being large.

\section{The deterministic phase}

\subsection{Outline}

In this section we show that the development of the proportion $L^{-1} V(t)$ of the volume of $C$ that is covered at time $t$ grows more or less deterministically, once the initial stages have passed, under assumption (2.25). As noted above, up to times of the form $\frac{1}{2} \lambda_{0}^{-1}\{\log \Lambda+x\}$ for fixed $x$, there are few self-intersections in the branching process $X^{*}$, so that, for such times, calculations made using the branching process can be expected to give close to the right answers for the small-world and gossip processes as well. Differences between $X_{P_{0}}^{*}$ and $Y_{P_{0}}$ arise because some islands in $X_{P_{0}}^{*}$ do not appear in $Y_{P_{0}}$, and are labelled as 'ghosts'; the $j$ th new branching process contact contributes to $Y$ (designated by $G_{j}=0$ ) only if it originates from a real (nonghost) island, and starts at a point outside $Y$, and multiple counting from overlapping islands is also prohibited.

The initial similarity between a small-world model and its branching process approximation was exploited in [4], when approximating the distribution of the distance $d_{\mathrm{SW}}\left(P, P^{\prime}\right)$ between two randomly chosen points $P$ and $P^{\prime}$ in the small-world graph. The key observation is that $d_{\mathrm{SW}}\left(P, P^{\prime}\right)>2 t$ exactly when the sets $Y_{P}(t)$ and $Y_{P^{\prime}}(t)$ are disjoint. The discussion above indicates that the sets $Y_{P}(t)$ and $Y_{P^{\prime}}(t)$ can be replaced with little error, for the calculation of probabilities, by the sets $Y_{P}^{*}(t)$ and $Y_{P^{\prime}}^{*}(t)$ generated by independent branching processes $X_{P}^{*}$ and $X_{P^{\prime}}^{*}$, as long as $t$ is of the form $\frac{1}{2} \lambda_{0}^{-1}\{\log \Lambda+x\}$, which is enough for our purposes. However, in small-world models, the argument is more complicated, because an intersection between an island $J$ of $X_{P}^{*}(t)$ and an island $J^{\prime}$ of $X_{P^{\prime}}^{*}(t)$ does not correspond to an intersection of $Y_{P}(t)$ with $Y_{P^{\prime}}(t)$ when either $J \subset J^{\prime}$ or $J^{\prime} \subset J$, since such a constellation cannot occur in the small-world process (see [4, Section 2, Type-II pairs]). Thus, for $P$ an independent uniform point of $C$, we have

$$
\mathbb{P}_{P_{0}}\left[d_{\mathrm{SW}}\left(P_{0}, P\right) \leq 2 t\right]=\mathbb{P}\left[Y_{P_{0}}(t) \cap Y_{P}(t) \neq \varnothing\right] \sim \mathbb{P}\left[Y_{P_{0}}^{*}(t) \cap^{*} Y_{P}^{*}(t) \neq \varnothing\right]
$$


for $Y_{P_{0}}^{*}(t)$ and $Y_{P}^{*}(t)$ generated by independent branching processes $X_{P_{0}}^{*}$ and $X_{P}^{*}$, where the notation ' $\cap$ *' is used to denote this special mode of intersection. The complement of the latter probability was approximated for small-world processes in [4, Theorem 4.2].

The connection with the current problem is that, in the small-world model,

$$
\mathbb{P}_{P_{0}}\left[d_{\mathrm{SW}}\left(P_{0}, P\right) \leq 2 t\right]=L^{-1} \mathbb{E} V_{P_{0}}(2 t),
$$

if $P$ is independently and uniformly chosen on $C$, and, similarly,

$$
L^{-1} \mathbb{E}\left\{V_{P_{0}}(2 t) \mid \mathcal{F}_{s}\right\}=\mathbb{P}_{P_{0}}\left[d_{\mathrm{SW}}\left(P_{0}, P\right) \leq 2 t \mid \mathcal{F}_{s}\right], \quad t>s,
$$

where $\mathcal{F}_{S}$ denotes the history of $Y_{P_{0}}$ up to time $s$. Then

$$
\mathbb{P}_{P_{0}}\left[d_{\mathrm{SW}}\left(P_{0}, P\right) \leq 2 t \mid \mathcal{F}_{s}\right] \sim \mathbb{P}\left[Y_{P_{0}}^{*}(t) \cap^{*} Y_{P}^{*}(t) \neq \varnothing \mid \mathcal{F}_{s}\right]=: \pi(s, t),
$$

where the asymptotic equivalence can be expected much as for (3.1). The aim is now to demonstrate that the quantity $L^{-1} V_{P_{0}}(2 t)$ stays close to its expectation $L^{-1} \mathbb{E}\left\{V_{P_{0}}(2 t) \mid \mathcal{F}_{s}\right\}$, showing that the volume process develops in almost deterministic fashion from $s$ onwards as long as $s$ is sufficiently large. For this, it is enough to show that the conditional variance $\operatorname{var}\left\{L^{-1} V_{P_{0}}(2 t) \mid \mathcal{F}_{s}\right\}$ becomes small with $s$. Now the expectation $L^{-1} \mathbb{E}\left\{V_{P_{0}}(2 t) \mid \mathcal{F}_{s}\right\}$ is approximated, as above, by $\pi(s, t)$. For the mean square, we simply note that, for $t>s$,

$$
L^{-2} \mathbb{E}\left\{\left[V_{P_{0}}(2 t)\right]^{2} \mid \mathcal{F}_{s}\right\}=\mathbb{P}_{P_{0}}\left[\left\{d_{\mathrm{SW}}\left(P_{0}, P\right) \leq 2 t\right\} \cap^{*}\left\{d_{\mathrm{SW}}\left(P_{0}, P^{\prime}\right) \leq 2 t\right\} \mid \widetilde{F}_{s}\right],
$$

where $P$ and $P^{\prime}$ are two independent uniform points of $C$, and then, again, much as for (3.1), we establish the approximation

$$
\begin{aligned}
\mathbb{P}_{P_{0}}\left[\left\{d_{\mathrm{SW}}\left(P_{0}, P\right) \leq 2 t\right\} \cap\left\{d_{\mathrm{SW}}\left(P_{0}, P^{\prime}\right) \leq 2 t\right\} \mid \mathcal{F}_{S}\right] \\
\quad \sim \mathbb{P}\left[\left\{Y_{P_{0}}^{*}(t) \cap^{*} Y_{P}^{*}(t) \neq \varnothing\right\} \cap\left\{Y_{P_{0}}^{*}(t) \cap^{*} Y_{P^{\prime}}^{*}(t) \neq \varnothing\right\} \mid \mathcal{F}_{s}^{*}\right],
\end{aligned}
$$

using three independent processes $X_{P_{0}}^{*}, X_{P}^{*}$, and $X_{P^{\prime}}^{*}$, where $\mathcal{F}_{s}^{*}$ denotes the history of $X_{P_{0}}^{*}$ up to $s$. Since, for large enough $s$, the development of the branching process $X_{P_{0}}^{*}$ after $s$ is almost deterministic, the statistics of the set $Y_{P_{0}}^{*}(t)$ are already almost determined at time $s$. Hence, the probability in (3.3) is close to

$$
\left\{\mathbb{P}\left[Y_{P_{0}}^{*}(t) \cap^{*} Y_{P}^{*}(t) \neq \varnothing \mid \mathcal{F}_{s}^{*}\right]\right\}^{2}=: \pi^{2}(s, t),
$$

asymptotically equivalent to the square of the conditional mean. Consequently, the conditional variance is small, as required. The remainder of the section consists of making an analogous argument precise, in the context of gossip processes. For small-world processes, the corresponding estimates can be deduced using the methods of [4, Section 4].

\subsection{Constructions}

For the gossip process, the following construction of $Y$ is useful. First, for any $(P, t) \in$ $C \times[0, \infty)$, let $S_{\mathcal{K}}(P, t) \subset C \times[0, \infty)$ denote the set whose $C$-section $\sigma_{C, u}\left\{S_{\mathcal{K}}(P, t)\right\}$ at $u$ is $\mathcal{K}(P, u-t)$ if $u \geq t$ and $\varnothing$ otherwise. Let $\Pi$ denote a marked Poisson process on $C \times[0, \infty)$ with constant intensity $\rho$, and with marks independently and uniformly distributed in $C$. Take $P_{0} \in C$ to be the initial point; set $S_{0}:=S_{\mathcal{K}}\left(P_{0}, 0\right)$. Then define $S_{1}$ to be the set

$$
S_{1}:=S_{0} \bigcup_{j \geq 1} S_{\mathcal{K}}\left(P_{1 j}, \tau_{1 j}\right),
$$


where the points of $\Pi$ in $S_{0}$ occur at locations and times $\left(Q_{1 j}, \tau_{1 j}\right)$ and have marks $P_{1 j}, j \geq 1$. Then, recursively, for $l \geq 2$, define

$$
S_{l}:=S_{l-1} \bigcup_{j \geq 1} S_{\mathcal{K}}\left(P_{l j}, \tau_{l j}\right),
$$

where the points of $\Pi$ in $S_{l-1} \backslash S_{l-2}$ occur at locations and times $\left(Q_{l j}, \tau_{l j}\right)$ and have marks $P_{l j}, j \geq 1$. Then we can define

$$
Y(t):=\sigma_{C, t}\left\{S_{\infty}\right\}, \quad 0 \leq t \leq T,
$$

where $S_{\infty}:=\bigcup_{l \geq 0} S_{l}$. Clearly, $S_{\infty} \cap\{C \times[0, T]\}=S_{l} \cap\{C \times[0, T]\}$ for some $l \geq 0$, since $\Pi$ has a.s. only finitely many points in $C \times[0, T]$.

Thinking of the points $\left(Q_{l j}, \tau_{l j}\right)$ with marks $P_{l j}$ as the $l$ th generation descendants of the initial individual $P_{0}$, we note that the path of the process $Y$ can be identified with that of a Markov branching process $X^{*}$ until the first time $t$ at which there is a nonempty intersection between two of the sets $S_{\mathcal{K}}\left(P_{l j}, t\right)$ for $l \geq 0$ and $j \geq 1$ such that $\tau_{l j} \leq t$; this is because of the independence of Poisson realizations on disjoint sets. Thus, the construction of $Y$ also yields a part of a coupled $X^{*}$.

As an alternative, the construction of $\Pi$ and $Y$ can be replicated starting from the branching process $X^{*}$. Start with $P_{0}$ and, writing $S_{0}:=S_{\mathcal{K}}\left(P_{0}, 0\right)$, assign Poisson points $\left(\tilde{Q}_{1 j}, \tilde{\tau}_{1 j}\right)$, $j \geq 1$, to $S_{0} \cap\{C \times[0, T]\}$ with intensity $\rho$, and with marks independently and uniformly distributed on $C$; denote these by $I_{1 j}:=\left(\tilde{Q}_{1 j}, \tilde{\tau}_{1 j} ; \tilde{P}_{1 j}\right), j \geq 1$. These are the first generation descendants of the individual at $P_{0}$ that are born before $T$. Repeat the process recursively, assigning at the $l$ th step, $l \geq 2$, Poisson points and marks to each $S_{l-1, j} \cap\{C \times[0, T]\}$, where $S_{l-1, j}:=S_{\mathcal{K}}\left(\tilde{P}_{l-1, j}, \tilde{\tau}_{l-1, j}\right)$, yielding the $l$ th generation descendants $I_{l j}:=\left(\tilde{Q}_{l j}, \tilde{\tau}_{l j} ; \tilde{P}_{l j}\right)$, $j \geq 1$, of the individual at $P_{0}$ that are born before $T$; as before, write $S_{l}:=S_{l-1} \cup\left\{\bigcup_{j \geq 1} S_{l j}\right\}$. There are in general more of these descendants than there are Poisson points and marks in $S_{\infty} \cap\{C \times[0, T]\}$, and the labelling is typically different. To recover the Poisson process $\Pi$ and its marks, first identify the set $\left\{I_{1 j}, j \geq 1\right\}$ with $\left\{\left(Q_{1 j}, \tau_{1 j} ; P_{1 j}\right), j \geq 1\right\}$, the first generation descendants of the individual at $P_{0}$ in the gossip process. Then label each $I_{1 j}$ with $G_{1 j} \in\{0,1\}$, according to whether the point is to be treated as real or a ghost; $G_{1 j}=1$ if $\left(P_{1 j}, \tau_{1 j}\right) \in S_{0}$, and, if there is any $j$ with $G_{1 j}=1$, the union in (3.4) is not disjoint beyond $\tau_{1 j}$.

For the remaining construction, the descendants $I_{l j ; r}$ of individual $I_{l j}$ in $[0, T]$ are the Poisson points and marks from $S_{l j} \cap\{C \times[0, T]\}$ chosen above. If $G_{l j}=1$, label them all with $G_{l j ; r}=1$-as descendants of ghosts, they are themselves ghosts. If $G_{l j}=0$, label those of the $I_{l j ; r}$ for which

$$
\left(\tilde{Q}_{l j ; r}, \tilde{\tau}_{l j ; r}\right) \in S_{l-1} \bigcup_{j^{\prime}=1}^{j-1} S_{l j^{\prime}}
$$

with $G_{l j ; r}=1$ also, since they lie in a part of $C \times[0, T]$ that has already been covered, and, therefore, do not belong to $\Pi$. Do the same if

$$
\left(\tilde{P}_{l j ; r}, \tilde{\tau}_{l j ; r}\right) \in S_{l-1} \bigcup_{j^{\prime}=1}^{j} S_{l j^{\prime}},
$$

since such points do not generate new descendants in $Y$, because then $S_{\mathcal{K}}\left(\tilde{P}_{l j ; r}, \tilde{\tau}_{l j ; r}\right)$ is contained in the already covered region of $C \times[0, \infty)$, even though here the points $\left(\tilde{Q}_{l j ; r}, \tilde{\tau}_{l j ; r}\right)$ do 
represent points of $\Pi$. The remaining points determine the points of $\Pi$ in $S_{l j} \backslash\left\{S_{l-1} \bigcup_{j^{\prime}=1}^{j-1} S_{l j^{\prime}}\right\}$ that carry the label $G_{l j ; r}=0$ and are in $C \times[0, T]$. The set of points

$$
\left\{\left(\tilde{Q}_{l j ; r}, \tilde{\tau}_{l j ; r}\right), j \geq 1, r \geq 1:\left(\tilde{Q}_{l j ; r}, \tilde{\tau}_{l j ; r}\right) \notin S_{l-1} \bigcup_{j^{\prime}=1}^{j-1} S_{l j^{\prime}}\right\},
$$

together with their marks $\tilde{P}_{l j ; r}$, can now be identified with the set of points and marks $\left\{\left(Q_{l+1, j}\right.\right.$, $\left.\left.\tau_{l+1, j} ; P_{l+1, j}\right), j \geq 1\right\}$, after a suitable reindexing (and with the labels $G_{l+1, j}$ correspondingly defined), recovering the gossip process $Y$.

Note that the same construction can also be used starting from two independent branching processes $X^{(1) *}$ and $X^{(2) *}$ with initial points $P_{0}^{(1)}$ and $P_{0}^{(2)}$, provided that, in each generation, the descendants of both individuals are listed together. The resulting gossip process describes the informed regions at each time $t$, when the information spreads from two initial sources at $P_{0}^{(1)}$ and $P_{0}^{(2)}$. Note that the order in which individuals appear in the list can influence the realization of $Y$ that is obtained, so that, for instance, the set $Y_{P_{0}^{(1)}}(t)$ obtained from $X^{(1) *}$ alone may not be identical to the subset of points within distance $t$ of $P_{0}^{(1)}$ in the set $Y_{P_{0}^{(1)}, P_{0}^{(2)}}(t)$ obtained from $X^{(1) *}$ and $X^{(2) *}$ together. However, it is shown in the proofs below that the differences, which only occur as a result of sets in the branching processes overlapping, are not significant for the ranges of $t$ under consideration here.

For the calculations to come, we need to show that analogues of the asymptotic equivalences in (3.2) and (3.3) hold for gossip processes. Here the argument is a little simpler than for small-world processes. A point $P$ has been informed from $P_{0}$ at time $2 t$ exactly when the set $Y_{P_{0}}(t) \subset C$ of points informed from $P_{0}$ by time $t$ intersects the set of points $\bar{Y}_{P}(t ; 2 t) \subset C$ from which the information will reach $P$ by time $2 t$ if it has reached $\bar{Y}_{P}(t ; 2 t)$ by time $t$. Now the set $\bar{Y}_{P}(t ; 2 t)$ is determined by the points $\left(\tau_{j}^{t}, P_{j}^{t}\right)$ of $\Pi$ in $C \times(t, 2 t]$, together with their associated marks $Q_{j}^{t}$, and can be constructed from them in exactly the same way as $Y_{P_{0}}(t)$ is constructed from the points $\left(\tau_{j}^{0}, P_{j}^{0}\right)$ of $\Pi$ in $C \times(0, t]$, together with their associated marks $Q_{j}^{0}$, except that time is run backwards from $2 t$ to $t$, and the roles of $P_{j}^{t}$ and $Q_{j}^{t}$ are swapped. Thus, and because the neighbourhoods $\mathcal{K}$ were chosen so that $P \in \mathcal{K}(Q, t)$ exactly when $Q \in \mathcal{K}(P, t)$, the set $\bar{Y}_{P}(t ; 2 t)$ has the same distribution as $Y_{P}(t)$, and $Y_{P_{0}}(t)$ and $\bar{Y}_{P}(t ; 2 t)$ are independent. Thus, we can deduce expressions based on which analogues of (3.2) and (3.3) can be justified, using the branching process approximations of Section 2: for any $s<t$,

$$
\begin{aligned}
& L^{-1} \mathbb{E}\left\{V_{P_{0}}(2 t) \mid \mathcal{F}_{s}\right\}=\mathbb{P}_{P_{0}}\left[Y_{P_{0}}(t) \cap \bar{Y}_{P}(t ; 2 t) \neq \varnothing \mid \mathcal{F}_{s}\right], \\
& L^{-2} \mathbb{E}\left\{\left[V_{P_{0}}(2 t)\right]^{2} \mid \mathcal{F}_{S}\right\} \\
& \quad=\mathbb{P}_{P_{0}}\left[\left\{Y_{P_{0}}(t) \cap \bar{Y}_{P}(t ; 2 t) \neq \varnothing\right\} \cap\left\{Y_{P_{0}}(t) \cap \bar{Y}_{P^{\prime}}(t ; 2 t) \neq \varnothing\right\} \mid \mathcal{F}_{S}\right],
\end{aligned}
$$

where $P$ and $P^{\prime}$ are independent uniform points of $C$. The argument is now primarily aimed at justifying the replacement of $Y_{P_{0}}(t), \bar{Y}_{P}(t ; 2 t)$, and $\bar{Y}_{P^{\prime}}(t ; 2 t)$ in the formulae above by independent copies of $Y_{Q}^{*}(t)$ with appropriate choices of $Q$, so that computations can be conveniently made.

\subsection{Calculations}

As remarked at the start of Section 2, it is useful to be able to bound $X^{*}$ above and below by branching processes having $c_{g}=0$, to which we can then apply the results of that section. We do this by constructing processes $X^{-}$and $X^{+}$, for times $t \geq s$, with the same initial conditions as $X^{*}$ at $s$, and using the same underlying Poisson process $Z$; the time $s$ and the initial conditions 
can be freely chosen. For $X^{*}$, the quantity $v(\mathcal{K}) M_{d}(u)$ has to be replaced by $V^{*}(u)$ in the argument of $Z$ in (2.5), and, by (2.25), we have

$$
v(\mathcal{K}) M_{d}(u)\left\{1-\eta_{\Lambda}\right\} \leq V^{*}(u) \leq v(\mathcal{K}) M_{d}(u)\left\{1+\eta_{\Lambda}\right\},
$$

where $\Lambda$ is as defined in (2.29) and

$$
\eta_{\Lambda}:=c_{g}\left(\frac{3 \log \Lambda}{2 \Lambda^{1 / d}}\right)^{\gamma_{g}}
$$

uniformly in $0 \leq u \leq \frac{3}{2} \lambda_{0}^{-1} \log \Lambda$. Hence, we can define $X^{+}$as in Section 2 by using $\rho^{+}:=$ $\rho\left\{1+\eta_{\Lambda}\right\}$ as the contact rate per unit volume, and $X^{-}$with $\rho^{-}:=\rho\left\{1-\eta_{\Lambda}\right\}$, in which case

$$
M_{l}^{-}(t) \leq M_{l}(t) \leq M_{l}^{+}(t) \quad \text { for all } 0 \leq t \leq \frac{3}{2} \lambda_{0}^{-1} \log \Lambda \text { and } 0 \leq l \leq d+1 .
$$

We shall use $X^{-}$and $X^{+}$extensively to bound quantities associated with $X^{*}$, and write

$$
\lambda_{0}^{ \pm}:=\lambda_{0}\left\{1 \pm \eta_{\Lambda}\right\}^{1 / d}
$$

for the corresponding growth exponents; for convenience, we shall assume henceforth that $\Lambda$ is large enough that $9 \eta_{\Lambda} \log \Lambda \leq 1$.

We now continue with the following Poisson approximation result (see [4, Theorem 3.1]), which can be simply proved using the Stein-Chen method.

Lemma 3.1. Let $n \mathcal{K}$-islands of radii $t_{1}, \ldots, t_{n} \leq \frac{3}{2} \lambda_{0}^{-1} \log \Lambda$ have centres independently and uniformly distributed on $C$, and let $N_{n}$ denote the number of pairs of them that intersect. Then

$$
d_{\mathrm{TV}}\left(\mathcal{L}\left(N_{n}\right), \operatorname{Po}\left(\mathbb{E} N_{n}\right)\right) \leq 4 n p_{\Lambda}^{+},
$$

where, recalling (2.26),

$$
p_{\Lambda}^{+}:=\frac{\{3 \log \Lambda\}^{d}}{\Lambda}\left\{1+c_{g}\left(\frac{3 \log \Lambda}{\Lambda^{1 / d}}\right)^{\gamma_{g}}\right\}=\frac{\{3 \log \Lambda\}^{d}}{\Lambda}\left\{1+2^{\gamma_{g}} \eta_{\Lambda}\right\}
$$

is an upper bound for the probability that two independently positioned $\mathcal{K}$-islands of radius at most $\frac{3}{2} \lambda_{0}^{-1} \log \Lambda$ intersect. Similarly, for two independent collections of $\mathcal{K}$-islands, one with $m$ and one with $n$ islands, having radii $t_{1}, \ldots, t_{m} \leq \frac{3}{2} \lambda_{0}^{-1} \log \Lambda$ and $u_{1}, \ldots, u_{n} \leq \frac{3}{2} \lambda_{0}^{-1} \log \Lambda$, respectively, the number $N_{m n}$ of intersecting pairs satisfies

$$
d_{\mathrm{TV}}\left(\mathcal{L}\left(N_{m n}\right), \operatorname{Po}\left(\mathbb{E} N_{m n}\right)\right) \leq 2(m+n) p_{\Lambda}^{+} .
$$

Thus, when finding the probability of there being an intersection between the islands of $X_{P_{1}}^{*}(t)$ and $X_{P_{2}}^{*}(t)$, where $X_{P_{1}}^{*}$ and $X_{P_{2}}^{*}$ are independent, this Poisson approximation offers an approach. It is exploited in the following result, in which $M^{(1)}$ and $M^{(2)}$ denote the quantities (2.2) derived from $X_{P_{1}}^{*}(t)$ and $X_{P_{2}}^{*}(t)$.

Lemma 3.2. Define

$$
P^{*}\left[m^{(1)}, m^{(2)}\right]:=\mathbb{P}\left[Y_{P_{1}}^{*}(t) \cap Y_{P_{2}}^{*}(t) \neq \varnothing \mid M^{(1)}(t)=m^{(1)}, M^{(2)}(t)=m^{(2)}\right] .
$$


Then, for $t \leq \frac{3}{2} \lambda_{0}^{-1} \log \Lambda$, we have

$$
\begin{aligned}
& \left|P^{*}\left[m^{(1)}, m^{(2)}\right]-\left(1-\exp \left\{-\sum_{j=0}^{d}\left(\begin{array}{l}
d \\
j
\end{array}\right) m_{j}^{(1)} m_{d-j}^{(2)} L^{-1} v(\mathcal{K})\right\}\right)\right| \\
& \leq 2\left(m_{0}^{(1)}+m_{0}^{(2)}\right) p_{\Lambda}^{+}+m_{0}^{(1)} m_{0}^{(2)} \frac{\{3 \log \Lambda\}^{d}}{\Lambda} 2^{\gamma_{g}} \eta_{\Lambda} .
\end{aligned}
$$

In particular, for $t=t_{\Lambda, x}:=\frac{1}{2} \lambda_{0}^{-1}\{\log \Lambda+x\}$, as in (2.30), $x \leq \frac{1}{2} \log \Lambda$, and $K \geq 1$, this gives

$$
\begin{gathered}
\left|P^{*}\left[M^{(1)}(t), M^{(2)}(t)\right]-\left(1-\exp \left\{-\sum_{j=0}^{d}\left(\begin{array}{l}
d \\
j
\end{array}\right) M_{j}^{(1)}(t) M_{d-j}^{(2)}(t) \lambda_{0}^{d} \Lambda^{-1}\right\}\right)\right| \\
\leq C\left\{K \Lambda^{-1 / 2+3 \varepsilon_{K} / 4}\{\log \Lambda\}^{d} \mathrm{e}^{x / 2}+K^{2} \Lambda^{-\gamma_{g} / d+3 \varepsilon_{K} / 2}\{\log \Lambda\}^{d+\gamma_{g}}\right\}
\end{gathered}
$$

for a suitable choice of $C$, except on an event of probability at most $2 c_{a} \mathrm{e}^{-K^{1 / 3} / 5}$.

Proof. The first part of the lemma is an immediate consequence of Lemma 3.1, together with (2.28). For the second part, we use (3.7) to bound $M_{0}^{(1)}(t)$ and $M_{0}^{(2)}(t)$ above by $M_{0}^{(1)+}(t)$ and $M_{0}^{(2)+}(t)$, and then Theorem 2.1(a) to show that, for $l=1,2$,

$$
M_{0}^{(l)}(t) \leq C_{a} K \mathrm{e}^{\lambda_{0}^{+} t\left(1+\varepsilon_{K}\right)} \leq \mathrm{e} C_{a} K \mathrm{e}^{\lambda_{0} t\left(1+\varepsilon_{K}\right)}
$$

for all $0 \leq t \leq \frac{3}{2} \lambda_{0}^{-1} \log \Lambda$, except on a set of probability at most $c_{a} \mathrm{e}^{-K^{1 / 3} / 5}$, since, for $t$ in this range,

$$
\lambda_{0}^{+} t\left(1+\varepsilon_{K}\right) \leq\left\{\lambda_{0} t+\frac{3}{2} \eta_{\Lambda} \log \Lambda\right\}\left(1+\varepsilon_{K}\right) \leq \lambda_{0} t\left(1+\varepsilon_{K}\right)+1,
$$

if $9 \eta_{\Lambda} \log \Lambda \leq 1$, since $\varepsilon_{K} \leq 5$ for $K \geq 1$ also.

We now aim to show that, for $t=t_{\Lambda, x}$ and $s<t$, the conditional probabilities of actual interest for (3.5),

$$
p_{1}(s, t):=\mathbb{P}_{P_{0}}\left[Y_{P_{0}}(t) \cap \bar{Y}_{P}(t ; 2 t) \neq \varnothing \mid \mathcal{F}_{S}\right]
$$

and

$$
p_{2}(s, t):=\mathbb{P}_{P_{0}}\left[\left\{Y_{P_{0}}(t) \cap \bar{Y}_{P}(t ; 2 t) \neq \varnothing\right\} \cap\left\{Y_{P_{0}}(t) \cap \bar{Y}_{P^{\prime}}(t ; 2 t) \neq \varnothing\right\} \mid \mathcal{F}_{s}\right]
$$

are close to the probabilities

$$
\begin{aligned}
& p_{1}^{*}(s, t):=\mathbb{E}_{P_{0}}\left\{P^{*}\left[M^{(1)}(t), M^{(2)}(t)\right] \mid \mathcal{F}_{s}\right\}, \\
& p_{2}^{*}(s, t):=\mathbb{E}_{P_{0}}\left\{P^{*}\left[M^{(1)}(t), M^{(2)}(t)\right] P^{*}\left[M^{(1)}(t), M^{(3)}(t)\right] \mid \mathcal{F}_{S}\right\},
\end{aligned}
$$

which we can approximate using Lemma 3.2. Here $M^{(1)}$ denotes the quantities (2.2) for $X_{P_{0}}^{*}$, coupled as above with $Y_{P_{0}}$, and $\mathcal{F}_{s}$ denotes its history up to $s ; M^{(2)}$ and $M^{(3)}$ are related to $\bar{Y}_{P}(t ; 2 t)$ and $\bar{Y}_{P^{\prime}}(t ; 2 t)$ in a similar fashion, through the branching processes $X_{P}^{*}$ and $X_{P^{\prime}}^{*}$, which are independent of each other and of $X_{P_{0}}^{*}$. Now the union $Y_{P_{0}}^{*}(t)$ of the islands of $X_{P_{0}}^{*}(t)$ contains $Y_{P_{0}}(t)$, and the corresponding statement is true for $X_{P}^{*}(t)$ and $\bar{Y}_{P}(t ; 2 t)$ and for $X_{P^{\prime}}^{*}(t)$ and $\bar{Y}_{P^{\prime}}(t ; 2 t)$. From this, it follows that $p_{1}^{*}(s, t) \geq p_{1}(s, t)$ and that $p_{2}^{*}(s, t) \geq p_{2}(s, t)$. We thus need to show only that the differences $p_{1}^{*}(s, t)-p_{1}(s, t)$ and $p_{2}^{*}(s, t)-p_{2}(s, t)$ cannot 
be too large. This we establish on the event $\tilde{A}_{K, s} \in \mathcal{F}_{s}$, defined as in (2.16) with $M_{i}^{(1)}(t) \lambda_{0}^{i} / i$ ! for $H_{i}(t)$, for a suitable choice of $K=K(\Lambda)$.

The differences between $p_{l}^{*}(s, t)$ and $p_{l}(s, t), l=1,2$, arise from events on which an island of a branching process $X^{*}$ makes an intersection that is not an intersection in the corresponding $Y$-process. Such an event can occur only if the island of $X^{*}$ is a ghost, or if the intersection occurs at a part of an island that overlaps another island, so that an intersection may have been counted twice using $X^{*}$. Thus, it will be enough to show that the probability of there being a ghost or an overlapped island in $X_{P_{0}}^{*}(t)$ that intersects $\bar{Y}_{P}(t ; 2 t) \cup \bar{Y}_{P^{\prime}}(t ; 2 t)$ is small, and that the same is true for ghosts and overlapped islands of $X_{P, P^{\prime}}^{*}(t):=X_{P}^{*}(t) \cup X_{P^{\prime}}^{*}(t)$ intersecting $Y_{P_{0}}(t)$.

Lemma 3.3. Define $K(\Lambda):=(40 \log \Lambda)^{3}$. Then, on $\tilde{A}_{K(\Lambda), s}$, for $t_{\Lambda, x}:=\left(2 \lambda_{0}\right)^{-1}\{\log \Lambda+x\}$ as in (2.30), and with $|x| \leq \frac{1}{6} \log \Lambda$,

$0 \leq p_{l}^{*}(s, t)-p_{l}(s, t)=O\left(\mathrm{e}^{x}\left(1+\mathrm{e}^{x}\right) \Lambda^{-5 / 12}\{\log \Lambda\}^{3 d+12}+\Lambda^{-1 / 6}\{\log \Lambda\}^{d+6}\right), \quad l=1,2$.

Proof. To make the necessary estimates, we begin by coupling $X_{P_{0}}^{*}$ in $t \geq s$ to upper and lower processes $X^{+}$and $X^{-}$as in (3.7), starting from the same state at time $s$. Define the event $A_{+}^{\prime}(K(\Lambda), s)$ as in (2.15), but for the process $X^{+}$, and so with $\lambda_{0}^{+}$for $\lambda_{0}$ and with $H^{+}$ for $H$. Then, on the event $A_{+}^{\prime}(K(\Lambda), s) \cap A_{\theta K, s}^{(1)}, M_{0}^{+}\left(t_{\Lambda, x}\right) \leq \mathrm{e} \theta K(\Lambda) \Lambda^{1 / 2} \mathrm{e}^{x / 2}$, where, in view of Theorem 2.1, $A_{+}^{\prime}(K(\Lambda), s)$ has conditional probability at least $1-c_{2} \Lambda^{-8}$ on $\tilde{A}_{K(\Lambda), s}$. Thus, the number $N\left(t_{\Lambda, x}\right)$ of intersecting pairs of islands of $X_{P_{0}}^{*}$ at $t_{\Lambda, x}$ satisfies

$$
\begin{aligned}
\mathbb{E}\left\{N\left(t_{\Lambda, x}\right) I\left[A_{+}^{\prime}(K(\Lambda), s)\right] \mid \mathcal{F}_{S} \cap \tilde{A}_{K(\Lambda), s}\right\} \\
\quad \leq \mathbb{E}\left\{\frac{1}{2}\left(M_{0}^{+}\left(t_{\Lambda, x}\right)\right)^{2} p_{\Lambda}^{+} I\left[A_{+}^{\prime}(K(\Lambda), s)\right] \mid \mathcal{F}_{S} \cap \tilde{A}_{K(\Lambda), s}\right\} \\
\quad \leq \frac{1}{2}\left\{C K(\Lambda) \Lambda^{1 / 2} \mathrm{e}^{x / 2}\right\}^{2} p_{\Lambda}^{+} \\
\quad \leq C^{\prime} \mathrm{e}^{x}\{\log \Lambda\}^{d+6}
\end{aligned}
$$

for some constants $C$ and $C^{\prime}$, using (3.9), and also

$$
4 \mathbb{E}\left\{M_{0}\left(t_{\Lambda, x}\right) I\left[A_{+}^{\prime}(K(\Lambda), s)\right] \mid \mathcal{F}_{s} \cap \tilde{A}_{K(\Lambda), s}\right\} p_{\Lambda}^{+} \leq C^{\prime \prime} \mathrm{e}^{x / 2} \Lambda^{-1 / 2}\{\log \Lambda\}^{d+3} .
$$

Thus, taking $x=-\frac{1}{6} \log \Lambda$, the conditional probability of any pair of islands of $X_{P_{0}}^{*}$ intersecting before time $\frac{5}{12} \lambda_{0}^{-1} \log \Lambda$ is at most of order $O\left(\Lambda^{-1 / 6}\{\log \Lambda\}^{d+6}\right)$ on $\tilde{A}_{K(\Lambda), s}$, from (3.10).

Now, for any fixed $C>0$, Bennett's inequality for the Poisson distribution implies that $\operatorname{Po}(m)\{[(m / C) \log \Lambda, \infty)\}=O\left(\Lambda^{-r}\right)$ for any $r \geq 0$, uniformly in $m \geq 1$, and a similar bound holds for $\operatorname{Po}(m)\{[\log \Lambda, \infty)\}$ uniformly in $0<m \leq 1$. Then, on $A_{+}^{\prime}(K(\Lambda), s)$,

$$
M_{0}\left(t_{\Lambda, x}\right) \leq M_{0}^{+}\left(t_{\Lambda, x}\right) \leq \mathrm{e} \theta K(\Lambda) \Lambda^{1 / 2} \mathrm{e}^{x / 2} .
$$

Thus, by Lemma 3.1 and (3.11), for any $r \geq 0$,

$$
\begin{aligned}
& \mathbb{P}\left[N\left(t_{\Lambda, x}\right)>\log \Lambda\left\{1 \vee \mathrm{e}^{x}\{\log \Lambda\}^{d+6}\right\} \mid \mathcal{F}_{s} \cap A_{+}^{\prime}(K(\Lambda), s) \cap \tilde{A}_{K(\Lambda), s}\right] \\
& \quad \leq C^{\prime \prime} \mathrm{e}^{x / 2} \Lambda^{-1 / 2}\{\log \Lambda\}^{d+3}+O\left(\Lambda^{-r}\right) \\
& \quad=O\left(\Lambda^{-1 / 4}\{\log \Lambda\}^{d+3}\right)
\end{aligned}
$$

if $x \leq \frac{1}{2} \log \Lambda$. Since $\mathbb{P}\left[A_{+}^{\prime}(K(\Lambda), s) \mid \tilde{A}_{K(\Lambda), s}\right] \geq 1-c_{2} \Lambda^{-8}$ also, the number of intersecting pairs of islands of $X_{P_{0}}^{*}$ before time $t_{\Lambda, x}$ exceeds $\log \Lambda\left\{1 \vee \mathrm{e}^{x}\{\log \Lambda\}^{d+6}\right\}$ with conditional probability of order $O\left(\Lambda^{-1 / 4}\{\log \Lambda\}^{d+3}\right)$. 
Thus, on $\tilde{A}_{K(\Lambda), s}$, except on an event of probability of order $O\left(\Lambda^{-1 / 6}\{\log \Lambda\}^{d+6}\right)$, all intersections of pairs of islands of $X_{P_{0}}^{*}$ occur after time $\frac{5}{12} \lambda_{0}^{-1} \log \Lambda$, and, once more in view of Theorem 2.1 applied to the dominating branching process $X^{+}$, they each give rise to at most $C(\log \Lambda)^{((d+1) \vee 3)} \Lambda^{1 / 12} \mathrm{e}^{x / 2}$ ghosts, except on an event of probability at most $c_{a} \Lambda^{-7}$, since each such intersection at worst gives rise to a ghost branching process starting with a single island of radius $\frac{3}{2} \lambda_{0}^{-1} \log \Lambda$. Thus, on $\tilde{A}_{K(\Lambda), s}$, except on an event of conditional probability of order $O\left(\Lambda^{-1 / 6}\{\log \Lambda\}^{d+6}\right)$, there are at most of order $O\left(\Lambda^{1 / 12}\{\log \Lambda\}^{2 d+9} \mathrm{e}^{x / 2}\left(1+\mathrm{e}^{x}\right)\right)$ islands in $X_{P_{0}}^{*}(t)$ whose intersections with $\bar{Y}_{P}(t ; 2 t) \cup \bar{Y}_{P^{\prime}}(t ; 2 t)$ should be discounted, and their radii cannot exceed $\frac{3}{2} \lambda_{0}^{-1} \log \Lambda$. Furthermore, the number of islands in $X_{P}^{*}(t)$ and $X_{P^{\prime}}^{*}(t)$ together is at most

$$
C_{a} K(\Lambda) \mathrm{e}^{\lambda_{0}^{+} t\left(1+\varepsilon_{K(\Lambda)}\right)}=O\left(\Lambda^{1 / 2}\{\log \Lambda\}^{3} \mathrm{e}^{x / 2}\right),
$$

except on an event of probability at most $c_{a} \Lambda^{-8}$, by Theorem 2.1 (a). Thus, on $\tilde{A}_{K(\Lambda), s}$, the mean number of intersections that should be neglected is, of the exceptional events, at most of order

$$
\begin{gathered}
O\left(\Lambda^{1 / 12}\{\log \Lambda\}^{2 d+9} \mathrm{e}^{x / 2}\left(1+\mathrm{e}^{x}\right) \Lambda^{1 / 2}\{\log \Lambda\}^{3} \mathrm{e}^{x / 2} p_{\Lambda}^{+}\right) \\
=O\left(\mathrm{e}^{x}\left(1+\mathrm{e}^{x}\right) \Lambda^{-5 / 12}\{\log \Lambda\}^{3 d+12}\right) .
\end{gathered}
$$

This expectation, together with the complementary expectation from overlapping islands and ghosts in $X_{P, P^{\prime}}^{*}$, bounds that part of the differences $p_{l}^{*}(s, t)-p_{l}(s, t), l=1,2$, arising off the exceptional events, and the exceptional events together have probability of order $O\left(\Lambda^{-1 / 6}\{\log \Lambda\}^{d+6}\right)$. The argument for this second expectation is the same, except that there is no conditioning, making it equivalent to the previous argument with $s=0$, and with $A(K(\Lambda), 0)$ being automatically satisfied because the initial state consists of just two singletons.

Lemma 3.4. For $M^{(1)}, M^{(2)}$, and $M^{(3)}$ as in Lemma 3.3, and for $x \leq \frac{1}{2} \log \Lambda$, we have

$$
\begin{gathered}
\mid \mathbb{E}_{(s)}\left\{\exp \left\{-\sum_{j=0}^{d}\left(\begin{array}{l}
d \\
j
\end{array}\right) M_{j}^{(1)}\left(t_{\Lambda, x}\right) M_{d-j}^{(2)}\left(t_{\Lambda, x}\right) \lambda_{0}^{d} \Lambda^{-1}\right\}\right\} \\
-\phi\left((d+1)^{-2} \sum_{j=0}^{d}\left(\begin{array}{l}
d \\
j
\end{array}\right) j !(d-j) ! W_{j}^{(1)}(s) \mathrm{e}^{x}\right) \mid \\
\leq C \mathrm{e}^{x}\left\{\mathrm{e}^{-\beta_{d+1} \lambda_{0} s}(\log \Lambda)^{3}+(\log \Lambda)^{4+\gamma_{g}} \Lambda^{-\gamma_{g} / d}\right\}
\end{gathered}
$$

and

$$
\begin{gathered}
\mid \mathbb{E}_{(s)}\left\{\exp \left\{-\sum_{j=0}^{d}\left(\begin{array}{l}
d \\
j
\end{array}\right) M_{j}^{(1)}\left(t_{\Lambda, x}\right)\left[M_{d-j}^{(2)}\left(t_{\Lambda, x}\right)+M_{d-j}^{(3)}\left(t_{\Lambda, x}\right)\right] \lambda_{0}^{d} \Lambda^{-1}\right\}\right\} \\
-\left\{\phi\left((d+1)^{-2} \sum_{j=0}^{d}\left(\begin{array}{c}
d \\
j
\end{array}\right) j !(d-j) ! W_{j}^{(1)}(s) \mathrm{e}^{x}\right)\right\}^{2} \mid \\
\leq C \mathrm{e}^{x}(\log \Lambda)^{6}\left\{\mathrm{e}^{-\beta_{d+1} \lambda_{0} s}+(\log \Lambda)^{1+\gamma_{g}} \Lambda^{-\gamma_{g} / d}\right\}
\end{gathered}
$$

for any $s \leq t_{\Lambda, x}$, where $\mathbb{E}_{(s)}$ denotes the expectation conditional on $\mathcal{F}_{s} \cap \tilde{A}_{K(\Lambda), s}, W_{j}^{(1)}(s):=$ $(d+1) \mathrm{e}^{-\lambda_{0} s} M_{j}^{(1)}(s) \lambda_{0}^{j} / j !, \phi$ is as in (2.21), and $C$ and $C^{\prime}$ are suitable constants. 
Proof. We give the proof of (3.13); that of (3.12) is simpler. For $t>s$, we bound $M^{(l)}$ above and below by $M^{(l)+}$ and $M^{(l)-}, 1 \leq l \leq 3$, as in the previous lemma. We then observe that, for $l=2,3$,

$$
\begin{aligned}
M_{j}^{(1)} & \left(t_{\Lambda, x}\right) M_{d-j}^{(l)}\left(t_{\Lambda, x}\right) \lambda_{0}^{d} \Lambda^{-1} \\
& \leq M_{j}^{(1)+}\left(t_{\Lambda, x}\right) M_{d-j}^{(l)+}\left(t_{\Lambda, x}\right)\left(\lambda_{0}^{+}\right)^{d} \Lambda^{-1} \\
& =(d+1)^{-2} j !(d-j) ! \mathrm{e}^{2 \lambda_{0}^{+} t} W_{j}^{(1)+}\left(t_{\Lambda, x}\right) W_{d-j}^{(l)+}\left(t_{\Lambda, x}\right) \Lambda^{-1} \\
& \leq(d+1)^{-2} j !(d-j) ! \mathrm{e}^{\left(1+\eta_{\Lambda}\right)(\log \Lambda+x)} W_{j}^{(1)+}\left(t_{\Lambda, x}\right) W_{d-j}^{(l)+}\left(t_{\Lambda, x}\right) \Lambda^{-1} \\
& \leq(d+1)^{-2} j !(d-j) ! \mathrm{e}^{x} W_{j}^{(1)+}\left(t_{\Lambda, x}\right) W_{d-j}^{(l)+}\left(t_{\Lambda, x}\right)\left\{1+2 \mathrm{e} \eta_{\Lambda} \log \Lambda\right\},
\end{aligned}
$$

where $\eta_{\Lambda}$ is as in (3.6). We now use Theorem 2.2(c) to deduce that

$$
\begin{aligned}
& \mathbb{E}_{(s)}\left(\left|W_{j}^{(1)+}\left(t_{\Lambda, x}\right)-W_{j}^{(1)+}(s)\right| I\left[A_{+}^{\prime}(K(\Lambda), s)\right]\right)=O\left(K(\Lambda) \mathrm{e}^{-\beta_{d+1} \lambda_{0}^{+} s}\right), \\
& \mathbb{E}_{(s)}\left(\left|W_{d-j}^{(l)+}\left(t_{\Lambda, x}\right)-W_{d-j}^{(l)+}(\infty)\right| I\left[A_{+}^{\prime}(K(\Lambda), s)\right]\right)=O\left(K(\Lambda) \mathrm{e}^{-\beta_{d+1} \lambda_{0}^{+} s}\right),
\end{aligned}
$$

for $l=2,3$, and Theorem 2.1(c) to show that $\mathbb{P}\left[A_{+}^{\prime}(K(\Lambda), s) \mid \mathcal{F}_{s} \cap \tilde{A}_{K(\Lambda), s}\right] \geq 1-c_{2} \Lambda^{-8}$. It thus follows, also using (2.20), that

$$
\begin{aligned}
& \mathbb{E}_{(s)}\left\{\exp \left\{-\sum_{j=0}^{d}\left(\begin{array}{c}
d \\
j
\end{array}\right) M_{j}^{(1)}\left(t_{\Lambda, x}\right)\left[M_{d-j}^{(2)}\left(t_{\Lambda, x}\right)+M_{d-j}^{(3)}\left(t_{\Lambda, x}\right)\right] \lambda_{0}^{d} \Lambda^{-1}\right\}\right\} \\
& \geq \mathbb{E}_{(s)}\left\{\exp \left\{-(d+1)^{-2} d ! \sum_{j=0}^{d} W_{j}^{(1)+}(s)\left[W_{*}^{(2)+}(\infty)+W_{*}^{(3)+}(\infty)\right] \mathrm{e}^{x}\right\}\right\} \\
&-O\left(\mathrm{e}^{x} K(\Lambda)^{2}\left\{\mathrm{e}^{-\beta_{d+1} \lambda_{0}^{+} s}+\eta_{\Lambda} \log \Lambda\right\}+\Lambda^{-8}\right) \\
&=\left\{\phi\left((d+1)^{-2} d ! \sum_{j=0}^{d} W_{j}^{(1)}(s) \mathrm{e}^{x}\right)\right\}^{2} \\
&-O\left(\mathrm{e}^{x}(\log \Lambda)^{6}\left[\mathrm{e}^{-\beta_{d+1} \lambda_{0} s}+(\log \Lambda)^{1+\gamma_{g}} \Lambda^{-\gamma_{g} / d}\right]\right),
\end{aligned}
$$

since $W_{j}^{(1)+}(s)=W_{j}^{(1)}(s)$ is $\mathcal{F}_{s}$-measurable, and since $W_{*}^{(2)+}(\infty)$ and $W_{*}^{(3)+}(\infty)$ are independent given $\mathcal{F}_{S}$, and each has the distribution of $W_{*}(\infty)$ as in (2.21). The upper bound is proved in an analogous fashion.

Theorem 3.1. Choose $s_{\Lambda}:=(\alpha / 2) \lambda_{0}^{-1} \log \Lambda$ for some $0<\alpha<\frac{1}{2}$. Let $\beta_{r}$ be as defined in Theorem 2.2, let $\tilde{A}_{K, s}$ be as for Lemma 3.3, and define $t_{\Lambda, x}$ as in (2.30). Then, for any $d$-dimensional gossip process satisfying (2.25) and any $\gamma_{1}<\gamma_{0}:=\min \left\{\frac{1}{3} \alpha \beta_{d+1}, \frac{2}{3} \gamma_{g} / d\right\}$, there exists a constant $k_{\gamma_{1}}<\infty$ such that

$$
\operatorname{var}\left\{L^{-1} V_{P_{0}}\left(2 t_{\Lambda, x}\right) \mid \mathcal{F}_{s_{\Lambda}} \cap \tilde{A}_{K(\Lambda), s_{\Lambda}}\right\} \leq k_{\gamma_{1}} \Lambda^{-\gamma_{1}},
$$

uniformly in $|x| \leq c_{v} \log \Lambda$, where $c_{v}:=\frac{1}{3}\left\{\frac{1}{2} \alpha \beta_{d+1} \wedge \gamma_{g} / d\right\}$. Furthermore, $\mathbb{P}\left[\tilde{A}_{K(\Lambda), s_{\Lambda}}\right] \geq$ $1-c_{A} \Lambda^{-\gamma_{2}}$ for some $\gamma_{2}>0$ and $c_{A}<\infty$. 
Proof. Starting from (3.5), collecting the results of Lemmas 3.2, 3.3, and 3.4, and taking $|x| \leq \frac{1}{8} \log \Lambda$, we find after comparing the various errors that

$$
\begin{aligned}
& \mathbb{E}\left\{L^{-1} V_{P_{0}}\left(2 t_{\Lambda, x}\right) \mid \mathcal{F}_{s_{\Lambda}} \cap \tilde{A}_{K(\Lambda), s_{\Lambda}}\right\} \\
&= G\left(W^{(1)}\left(s_{\Lambda}\right), x\right) \\
&+O\left(\mathrm{e}^{x}\left\{(\log \Lambda)^{3} \Lambda^{-\alpha \beta_{d+1} / 2}+(\log \Lambda)^{4+\gamma_{g}} \Lambda^{-\gamma_{g} / d}\right\}+(\log \Lambda)^{d+6+\gamma_{g}} \Lambda^{-\gamma_{d} / d}\right) \\
&= G\left(W^{(1)}\left(s_{\Lambda}\right), x\right)+O\left(\mathrm{e}^{x}(\log \Lambda)^{4+\gamma_{g}} \Lambda^{-3 c_{v}}+(\log \Lambda)^{d+6+\gamma_{g}} \Lambda^{-\gamma_{g} / d}\right),
\end{aligned}
$$

where

$$
G(w, x)=1-\phi_{d}\left(\mathrm{e}^{x}(d+1)^{-2} d ! \sum_{j=0}^{d} w_{j}\right)
$$

Similarly,

$$
\begin{aligned}
\mathbb{E}\left\{\left[L^{-1} V_{P_{0}}\left(2 t_{\Lambda, x}\right)\right]^{2} \mid \mathcal{F}_{s_{\Lambda}} \cap \tilde{A}_{K(\Lambda), s_{\Lambda}}\right\} \\
\quad=\left\{G\left(W^{(1)}\left(s_{\Lambda}\right), x\right)\right\}^{2}+O\left(\mathrm{e}^{x}(\log \Lambda)^{4+\gamma_{g}} \Lambda^{-3 c_{v}}+(\log \Lambda)^{d+6+\gamma_{g}} \Lambda^{-\gamma_{g} / d}\right) .
\end{aligned}
$$

Combining (3.14) and (3.15), and bounding the error term, the first statement of the theorem follows.

To bound $\mathbb{P}\left[\tilde{A}_{K(\Lambda), s_{\Lambda}}\right]$ from below, we start with Theorem 2.1(a). Putting $s=0$ and then $t=s_{\Lambda}$, and setting $K=K(\Lambda)=(40 \log \Lambda)^{3}$, it follows that

$$
\mathbb{P}\left[\mathrm{e}^{-\lambda_{0} s_{\Lambda}}\left\|H\left(s_{\Lambda}\right)\right\| \leq C_{a} K(\Lambda) \mathrm{e}^{1 / 80}\right] \geq 1-c_{a} \Lambda^{-8},
$$

so that $\mathbb{P}\left[A_{\theta K(\Lambda), s_{\Lambda}}^{(1)}\right] \geq 1-c_{a} \Lambda^{-8}$ if $\theta:=C_{a} \mathrm{e}^{1 / 80}$. Then, from Lemma 2.2,

$$
\mathbb{P}\left[A_{K(\Lambda), s_{\Lambda}}^{(2)}\right]=\mathbb{P}\left[H_{r(d)}\left(s_{\Lambda}\right) \geq K(\Lambda)\right]=\mathbb{P}\left[\tau_{K(\Lambda)}^{r(d)} \leq s_{\Lambda}\right],
$$

bounded below by $1-2 K(\Lambda) \exp \left\{-s_{\Lambda} \lambda_{0} / c_{c}\right\}=1-C \Lambda^{-\gamma}$ for some $\gamma>0$, because of the choice of $s_{\Lambda}$. Finally, from Lemma $2.1(\mathrm{~d})$, since $K(\Lambda)^{\left(1-\varepsilon_{K(\Lambda)}\right) / 2}\left(2^{\varepsilon_{K(\Lambda)}}-1\right) \asymp(\log \Lambda)^{1 / 2}$ exceeds $42 \log 2$ for all sufficiently large $\Lambda$,

$$
1-\mathbb{P}\left[A_{K(\Lambda), \varepsilon_{K(\Lambda)}, s_{\Lambda}}^{(3)}\right] \leq c_{4} \exp \left\{-\frac{1}{28} K(\Lambda)^{\left(1-\varepsilon_{K(\Lambda)}\right) / 2}\right\} \leq C_{1} \exp \left\{-C_{2}(\log \Lambda)^{3 / 2}\right\},
$$

completing the proof.

Theorem 3.1 is the basis for the main result of the paper, showing that the distribution of the path $L^{-1} V_{P_{0}}(t)$ is concentrated close to its conditional mean. To complete the proof, we first need to have an expression for the conditional mean.

Lemma 3.5. Uniformly in $|x| \leq c_{v} \log \Lambda$, and with $s_{\Lambda}:=(\alpha / 2) \lambda_{0}^{-1} \log \Lambda$ for some $0<$ $\alpha<\frac{1}{2}$, $\mathbb{E}\left\{L^{-1} V_{P_{0}}\left(\lambda_{0}^{-1}\{\log \Lambda+x\}\right) \mid \mathcal{F}_{S_{\Lambda}} \cap \tilde{A}_{K(\Lambda), s_{\Lambda}}\right\}=h_{d}\left(x+\log C_{d}+\log W_{*}^{(1)}\left(s_{\Lambda}\right)\right)+O\left(\Lambda^{-\gamma_{1}}\right)$, where $h_{d}$ is as in (2.23), $C_{d}:=(d+1)^{-1} d$ !, and $\gamma_{1}$ is as for Theorem 3.1. Also, if $P$ is independently and uniformly chosen on $C$, the time $\tau_{P}:=\inf \left\{t \geq 0: P \in Y_{P_{0}}(t)\right\}$ satisfies

$$
\mathbb{P}\left[\tau_{P}>\lambda_{0}^{-1}(\log \Lambda+x)\right]=\mathbb{E}\left(\exp \left\{-\mathrm{e}^{x} C_{d} W_{*}^{(1)}(\infty) W_{*}^{(2)}(\infty)\right\}\right)+O\left(\Lambda^{-\gamma_{3}}\right)
$$

for some $\gamma_{3}>0$, where $W_{*}^{(1)}(\infty)$ and $W_{*}^{(2)}(\infty)$ are independent. 
Proof. By arguing as for Lemma 3.4, using Theorem 2.2(c), it follows that, uniformly for $x$ as in Theorem 3.1,

$$
\begin{aligned}
\mathbb{E}\left\{L^{-1} V_{P_{0}}\left(2 t_{\Lambda, x}\right) \mid \mathcal{F}_{s_{\Lambda}} \cap \tilde{A}_{K(\Lambda), s_{\Lambda}}\right\} & =\mathbb{E}\left\{G\left(W^{(1)}\left(s_{\Lambda}\right), x\right) \mid \mathcal{F}_{s_{\Lambda}} \cap \tilde{A}_{K(\Lambda), s_{\Lambda}}\right\}+O\left(\Lambda^{-\gamma_{1}}\right) \\
& =\mathbb{E}\left\{G\left(W_{*}^{(1)}\left(s_{\Lambda}\right) \mathbf{1}, x\right) \mid \mathcal{F}_{S_{\Lambda}} \cap \tilde{A}_{K(\Lambda), s_{\Lambda}}\right\}+O\left(\Lambda^{-\gamma_{1}}\right) \\
& =1-\phi_{d}\left(C_{d} \mathrm{e}^{x} W_{*}^{(1)}\left(s_{\Lambda}\right)\right)+O\left(\Lambda^{-\gamma_{1}}\right),
\end{aligned}
$$

where $C_{d}$ is as defined above, and the first part follows from the definition of $h_{d}$ following (2.21). The final result follows from taking the unconditional expectation in (3.16) and applying Theorem 2.2(c):

$$
\begin{aligned}
1-\mathbb{E}\left\{L^{-1} V_{P_{0}}\left(2 t_{\Lambda, x}\right)\right\} & =\mathbb{E}\left\{\phi_{d}\left(C_{d} \mathrm{e}^{x} W_{*}^{(1)}\left(s_{\Lambda}\right)\right)\right\}+O\left(\Lambda^{-\gamma_{1}}\right)+O\left(\Lambda^{-\gamma_{2}}\right) \\
& =\mathbb{E}\left\{\phi_{d}\left(C_{d} \mathrm{e}^{x} W_{*}^{(1)}(\infty)\right)\right\}+O\left(\Lambda^{-\gamma_{3}}\right)
\end{aligned}
$$

for some $\gamma_{3}>0$.

Note that the form of the neighbourhoods $\mathcal{K}$ only comes into the formulae of Lemma 3.5 through their volume $v(\mathcal{K})$, which is implicitly present in the time scaling by $\lambda_{0}$ in the definition of $t_{\Lambda, x}$.

We are now in a position to prove the pathwise approximation to $L^{-1} V_{P_{0}}(t)$. Before doing so, we note that $h_{d}$ is the distribution function of a sum of independent random variables $Z_{1}$ and $Z_{2}$, where $-Z_{1}$ has a standard Gumbel distribution, and $Z_{2}$ is distributed as $-\log W_{*}(\infty)$. This is because $h_{d}(x):=1-\phi_{d}\left(\mathrm{e}^{x}\right)$ can be rewritten in alternative form as

$$
1-\mathbb{E}\left\{\mathrm{e}^{-\mathrm{e}^{Z}}\right\}=\mathbb{P}\left[-Z_{1} \geq-Z\right]=\mathbb{P}\left[Z_{1}+Z_{2} \leq x\right],
$$

where $Z:=x+\log W_{*}(\infty)$ is independent of $Z_{1}$.

Theorem 3.2. For any d-dimensional gossip process satisfying (2.25), there exists a random variable $U$ such that

$$
\mathbb{P}\left[\sup _{x}\left|L^{-1} V_{P_{0}}\left(\lambda_{0}^{-1}\{\log \Lambda+x\}\right)-h_{d}\left(x+\log C_{d}+U\right)\right|>4 \Lambda^{-a_{1}}\right] \leq c \Lambda^{-a_{2}}
$$

for some positive $a_{1}, a_{2}$ and $c<\infty$. Here $\mathrm{e}^{U}$ has the distribution of $W_{*}^{[d+1]}(\infty)$ as in Remark 2.2, whose Laplace transform satisfies (2.22), $C_{d}$ is as defined in Lemma 3.5, $h_{d}$ is as in (2.23), and $\Lambda$ is as defined in (2.29).

Proof. Take $s_{\Lambda}:=(\alpha / 2) \lambda_{0}^{-1} \log \Lambda$ for some $0<\alpha<\frac{1}{2}$. It then follows from Theorem 3.1, Lemma 3.5, and Chebyshev's inequality that, for any $a<\gamma_{1} / 2$,

$$
\begin{aligned}
& \mathbb{P}\left[\left|L^{-1} V_{P_{0}}\left(\lambda_{0}^{-1}\{\log \Lambda+x\}\right)-h_{d}\left(x+\log C_{d}+\log W_{*}^{(1)}\left(s_{\Lambda}\right)\right)\right|>2 \Lambda^{-a} \mid \mathcal{F}_{s_{\Lambda}} \cap \tilde{A}_{K(\Lambda), s_{\Lambda}}\right] \\
& \quad \leq k_{\gamma_{1}} \Lambda^{2 a-\gamma_{1}}
\end{aligned}
$$

uniformly for $|x| \leq c_{v} \log \Lambda$.

Now, representing $h$ using $Z_{1}$ and $Z_{2}$ as above, we have, for $x>0$,

$$
\begin{gathered}
h_{d}(-x) \leq \mathbb{P}\left[-Z_{1} \geq \frac{1}{2} x\right]+\mathbb{P}\left[\log W_{*}(\infty) \geq \frac{1}{2} x\right] \leq 2 \mathrm{e}^{-x / 2}, \\
1-h_{d}(x) \leq \mathbb{P}\left[-Z_{1} \leq-\frac{1}{2} x\right]+\mathbb{P}\left[\log W_{*}(\infty) \leq-\frac{1}{2} x\right] \leq \exp \left\{-\mathrm{e}^{x / 2}\right\}+\varepsilon(x),
\end{gathered}
$$


where $\varepsilon(x)$ goes to 0 superexponentially fast, by (2.24). Thus, for $x_{\Lambda}^{+}:=\frac{1}{2} c_{v} \log \Lambda$, $1-h_{d}\left(x_{\Lambda}^{+}\right)=O\left(\Lambda^{-1}\right)$, and, for $x_{\Lambda}^{-}:=-\frac{1}{2} c_{v} \log \Lambda, h_{d}\left(x_{\Lambda}^{-}\right) \leq 2 \Lambda^{-c_{v} / 4}$. Now choose an increasing sequence of $m_{\Lambda}:=\left\lceil\Lambda^{b}\right\rceil$ points $x_{\Lambda}^{(j)}$ between $x_{\Lambda}^{-}$and $x_{\Lambda}^{+}$, with $x_{\Lambda}^{(0)}=x_{\Lambda}^{-}$ and $x_{\Lambda}^{\left(m_{\Lambda}\right)}=x_{\Lambda}^{+}$, in such a way that $h_{d}\left(x_{\Lambda}^{(j+1)}\right)-h_{d}\left(x_{\Lambda}^{(j)}\right) \leq \Lambda^{-b}$ for each $j$. On $\tilde{A}_{K(\Lambda), s_{\Lambda}}$, from (2.14),

$$
-\log W_{*}^{(1)}\left(s_{\Lambda}\right) \leq \lambda_{0} s_{\Lambda}=\frac{1}{2} \alpha \log \Lambda, \quad \log W_{*}^{(1)}\left(s_{\Lambda}\right) \leq \log (d K(\Lambda)),
$$

so that, choosing $\alpha \leq \frac{1}{2} c_{v},\left|\log W_{*}^{(1)}\left(s_{\Lambda}\right)\right| \leq \frac{1}{4} c_{v} \log \Lambda$ for all large enough $\Lambda$. Thus, we can take $x=x_{\Lambda}^{(j)}-\log W_{*}^{(1)}\left(s_{\Lambda}\right)$ in (3.17) for each $0 \leq j \leq m_{\Lambda}$, obtaining

$$
\begin{aligned}
& \mathbb{P}\left[\max _{0 \leq j \leq m_{\Lambda}}\left|L^{-1} V_{P_{0}}\left(\lambda_{0}^{-1}\left\{\log \Lambda+x_{\Lambda}^{(j)}-\log W_{*}^{(1)}\left(s_{\Lambda}\right)-\log C_{d}\right\}\right)-h_{d}\left(x_{\Lambda}^{(j)}\right)\right|\right. \\
& \left.>2 \Lambda^{-a} \mid \mathcal{F}_{s_{\Lambda}} \cap \tilde{A}_{K(\Lambda), s_{\Lambda}}\right] \\
& \quad \leq 2 k_{\gamma_{1}} \Lambda^{2 a+b-\gamma_{1}},
\end{aligned}
$$

from which it follows directly, because both $V_{P_{0}}(t)$ and $h_{d}(x)$ are nondecreasing in their arguments, that

$$
\begin{gathered}
\mathbb{P}\left[\sup _{x}\left|L^{-1} V_{P_{0}}\left(\lambda_{0}^{-1}\{\log \Lambda+x\}\right)-h_{d}\left(x+\log C_{d}+\log W_{*}^{(1)}\left(s_{\Lambda}\right)\right)\right|\right. \\
\left.>2 \Lambda^{-a}+\Lambda^{-b} \mid \mathcal{F}_{s_{\Lambda}} \cap \tilde{A}_{K(\Lambda), s_{\Lambda}}\right] \\
\quad \leq k \Lambda^{2 a+b-\gamma_{1}}
\end{gathered}
$$

for a suitable constant $k$.

The above estimate almost completes the theorem; it remains to replace $W_{*}^{(1)}\left(s_{\Lambda}\right)$ by $W_{*}^{(1)+}(\infty)$, where $W^{(1)+}$ is as in Lemma 3.3, and then to remove the conditioning. For the former, we have

$$
\begin{aligned}
& \mathbb{E}\left\{\left|W_{*}^{(1)}\left(s_{\Lambda}\right)-W_{*}^{(1)+}(\infty)\right| I\left[A_{+}^{\prime}\left(K(\Lambda), s_{\Lambda}\right)\right] \mid \mathcal{F}_{s_{\Lambda}} \cap \tilde{A}_{K(\Lambda), s_{\Lambda}}\right\} \\
& =O\left(K(\Lambda) \exp \left\{-\beta_{d+1} \lambda_{0}^{+} s_{\Lambda}\right\}\right)
\end{aligned}
$$

from Theorem 2.2(c), together with $\mathbb{P}\left[A_{+}^{\prime}\left(K(\Lambda), s_{\Lambda}\right) \mid \mathcal{F}_{s_{\Lambda}} \cap \tilde{A}_{K(\Lambda), s_{\Lambda}}\right] \geq 1-c_{2} \Lambda^{-8}$ from Theorem 2.1(b). Therefore,

$$
\mathbb{P}\left[\left|W_{*}^{(1)}\left(s_{\Lambda}\right)-W_{*}^{(1)+}(\infty)\right|>\Lambda^{-b} \mid \mathcal{F}_{s_{\Lambda}} \cap \tilde{A}_{K(\Lambda), s_{\Lambda}}\right]=O\left(\Lambda^{-b^{\prime}}\right)
$$

for any $b^{\prime}<\frac{1}{2} \beta_{d+1} \alpha-b$, and, thus, for any $b^{\prime}<3 c_{v}-b$, and, hence, also

$$
\begin{aligned}
& \mathbb{P}\left[\left|\log W_{*}^{(1)}\left(s_{\Lambda}\right)-\log W_{*}^{(1)+}(\infty)\right|>\Lambda^{-b / 2} \mid \mathcal{F}_{s_{\Lambda}} \cap \tilde{A}_{K(\Lambda), s_{\Lambda}} \cap\left\{W_{*}^{(1)+}(\infty) \geq \Lambda^{-b / 2}\right\}\right] \\
& =O\left(\Lambda^{-b^{\prime}}\right) .
\end{aligned}
$$

Since $h_{d}$ has a density bounded by $1 / \mathrm{e}$, the maximum of the density of the Gumbel distribution, it follows that

$$
\begin{aligned}
& \mathbb{P}\left[\sup _{x}\left|L^{-1} V_{P_{0}}\left(\lambda_{0}^{-1}\{\log \Lambda+x\}\right)-h_{d}\left(x+\log C_{d}+\log W_{*}^{(1)+}(\infty)\right)\right|\right. \\
& \left.>\left(2 \Lambda^{-a}+\Lambda^{-b}+\mathrm{e}^{-1} \Lambda^{-b / 2}\right) \mid \mathcal{F}_{s_{\Lambda}} \cap \tilde{A}_{K(\Lambda), s_{\Lambda}} \cap\left\{W_{*}^{(1)+}(\infty) \geq \Lambda^{-b / 2}\right\}\right] \\
& \quad \leq k \Lambda^{2 a+b-\gamma_{1}}+k^{\prime} \Lambda^{-b^{\prime}} .
\end{aligned}
$$


But now, from (2.24), $\mathbb{P}\left[W_{*}^{(1)+}(\infty)<\Lambda^{-b / 2}\right]=o\left(\Lambda^{-u}\right)$ for any $u>0$, and $\mathbb{P}\left[\tilde{A}_{K(\Lambda), s_{\Lambda}}\right] \geq$ $1-c_{A} \Lambda^{-\gamma_{2}}$ from Theorem 3.1, so that

$$
\begin{gathered}
\mathbb{P}\left[\sup _{x}\left|L^{-1} V_{P_{0}}\left(\lambda_{0}^{-1}\{\log \Lambda+x\}\right)-h_{d}\left(x+\log C_{d}+\log W_{*}^{(1)+}(\infty)\right)\right|\right. \\
\left.>2 \Lambda^{-a}+\Lambda^{-b}+\mathrm{e}^{-1} \Lambda^{-b / 2}\right] \\
\quad \leq k \Lambda^{2 a+b-\gamma_{1}}+c_{A} \Lambda^{-\gamma_{2}}+c^{\prime} \Lambda^{-b^{\prime}} .
\end{gathered}
$$

Now take $2 a=b=\left(c_{v} \wedge\left\{\gamma_{1} / 4\right\}\right)$ and $b^{\prime}=c_{v}$ to complete the proof, with $a_{1}=b / 2$ and $a_{2}=\min \left\{\gamma_{1} / 2, \gamma_{2}, c_{v}\right\}$.

For small-world processes, the counterpart of Theorem 3.1 can be proved in an entirely similar fashion. Lemma 3.5 is also correct, if $C_{d}$ is replaced by

$$
\tilde{C}_{d}:=d^{-2} d !\{(d+1)-1\}=(d-1) !
$$

the change reflects both the difference in $r(d)$ between gossip and small-world processes, and the subtracted component arising from intersections forbidden in the small-world context; see [4, Section 4] for more details. Note that the expression in [4, Theorem 4.2] appears different from that obtained here. It is, however, the same, with the transformations $r \rightarrow d, 2 x \rightarrow x$, $\alpha_{l} \rightarrow v(\mathcal{K})\left(\begin{array}{l}d \\ l\end{array}\right)$, and $W \rightarrow d^{-1} W_{*}(\infty)$, and noting that $L \rho=\Lambda / d$ !. For the limiting random variable $W_{*}(\infty)$, the Poisson process of descendants of the first individual now has intensity

$$
\rho v(\mathcal{K}) d u^{d-1}=\frac{\lambda_{0}^{d} u^{d-1}}{(d-1) !}, \quad u>0,
$$

giving

$$
\phi(\theta)=\exp \left\{-\int_{0}^{\infty} \frac{1}{(d-1) !}\left\{1-\phi\left(\theta \mathrm{e}^{-x}\right)\right\} x^{d-1} \mathrm{~d} x\right\},
$$

the same equation as (2.22) for gossip processes, except that now $d$ is replaced by $d-1$; in view of (2.8), this is not surprising. We thus have the corresponding law of large numbers approximation.

Theorem 3.3. For any d-dimensional small-world process satisfying (2.25), there exists a random variable $\tilde{U}$ such that

$$
\mathbb{P}\left[\sup _{x}\left|L^{-1} V_{P_{0}}\left(\lambda_{0}^{-1}\{\log \Lambda+x\}\right)-h_{d-1}\left(x+\log \tilde{C}_{d}+\tilde{U}\right)\right|>4 \Lambda^{-\tilde{a}_{1}}\right] \leq \tilde{c} \Lambda^{-\tilde{a}_{2}}
$$

for some positive $\tilde{a}_{1}, \tilde{a}_{2}$ and $\tilde{c}<\infty$. Here $\mathrm{e}^{\tilde{U}}$ has the distribution of $W_{*}^{[d]}(\infty)$ as defined in Remark 2.2, $\tilde{C}_{d}$ is as defined in (3.18), $h_{r}$ is as in (2.23), and $\Lambda$ is as defined in (2.29).

For the gossip process studied by Chatterjee and Durrett [6], $C$ is an $N \times N$ torus, so that $d=2$ and $L=N^{2}$, and $\mathcal{K}(P, s)=B(P, s / \sqrt{2 \pi})$ is a Euclidean ball, so that $v(\mathcal{K})=\frac{1}{2}$ and $c_{g}=0$. They set $\rho=N^{-\alpha}$ for any $\alpha<3$, so that $\lambda_{0}=(d ! \rho v(\mathcal{K}))^{1 /(d+1)}=N^{-\alpha / 3}$ and $\Lambda=L \lambda_{0}^{d} / v(\mathcal{K})=2 N^{2(1-\alpha / 3)}$; also, $C_{d}=\frac{2}{3}$ in Lemma 3.5. Then the pathwise approximation given in Theorem 3.2 matches $N^{-2} V\left(N^{\alpha / 3}[2(1-\alpha / 3) \log N+x+\log 2]\right)$ with $h\left(x+\log \left(\frac{2}{3}\right)+U\right)$. This is seen to be the same as in Chatterjee and Durrett [6], noting that $U$ has the same distribution as their $\log M$, and that our choice of $h(\cdot)$ as a solution of (2.23) corresponds to their $h(\cdot+\log 3)$, since they implicitly chose the solution of $(2.23)$ that has $\lim _{t \rightarrow-\infty} \mathrm{e}^{-t} h^{\prime}(t)=\frac{1}{3}$. 


\subsection{Complete coverage}

Theorem 3.2 and (3.16), together with the fact that $2 t_{\Lambda, x}=\lambda_{0}^{-1}\{\log \Lambda+x\}$, show that only a negligible fraction of $C$ is covered at times much before $\lambda_{0}^{-1} \log \Lambda$, with $C$ then becoming essentially covered in a deterministic fashion on a time scale of order $O(1)$ around $\lambda_{0}^{-1} \log \Lambda$. Randomness is visible only in the time shift of $-\lambda_{0}^{-1} U$ in the origin of the transition from uncovered to covered.

One can also, as in Chatterjee and Durrett [6], consider how long it takes until $C$ is entirely covered. We show that complete coverage is achieved relatively soon after time $\lambda_{0}^{-1} \log \Lambda$, under the assumption that, for each $s, C$ can be covered by $n(s)$ islands of the form $\mathcal{K}(P, s)$, where $n(s)$ satisfies

$$
n(s) \leq \frac{c_{0} L}{v(\mathcal{K}) s^{d}}, \quad 0<s<L^{1 / d},
$$

for some $c_{0}$.

Theorem 3.4. If (3.19) is satisfied for a d-dimensional gossip process satisfying (2.25) then, except on a set of probability of order $O\left(\Lambda^{-\delta}\right)$ for some $\delta>0$, the whole of $C$ is covered before time

$$
\tau(\Lambda, s)+\frac{2}{\lambda_{0}}\left\{\frac{72 \log \Lambda}{d !}\right\}^{1 / d},
$$

where $\tau(\Lambda, s)=\lambda_{0}^{-1}\left\{\log \Lambda+O\left((\log \Lambda)^{1 /(d+1)}\right)\right\}$. The corresponding result holds for a smallworld process, now with $\tau(\Lambda, s)=\lambda_{0}^{-1}\left\{\log \Lambda+O\left((\log \Lambda)^{1 / d}\right)\right\}$.

Proof. In a gossip process, in view of Theorem 3.2, at least $\frac{1}{4} \Lambda / d$ ! contacts have been made up to time $\tau(\Lambda, s):=\lambda_{0}^{-1}\left\{\log \Lambda+\mathfrak{m}-U-\log C_{d}\right\}+\lambda_{0}^{-1}$, with probability $1-O\left(\Lambda^{-\delta}\right)$ for some $\delta>0$, where $\mathfrak{m}$ denotes the median $h_{d}^{-1}\left(\frac{1}{2}\right)$; this is because, at this time, a volume of about $L / 2$ has been generating contacts for a time interval of at least $\lambda_{0}^{-1}$. Write $\psi:=d ! / 4$, and also note that, from $(2.24),-U \leq(k \log \Lambda)^{1 /(d+1)}$ except on an event of probability of order $O\left(\Lambda^{-8}\right)$, if $k$ is chosen large enough.

Cover $C$ with islands of radius $s=k \lambda_{0}^{-1}$; with $c_{0}$ as in (3.19) this can be done using at most $c_{0} L /\left\{v(\mathcal{K})\left(k / \lambda_{0}\right)^{d}\right\}$ islands. Then, recalling (2.25), the probability that any of these islands contains none of $P_{1}, \ldots, P_{\lceil\psi \Lambda\rceil}$ is at most

$$
\frac{c_{0} v(\mathcal{K}) \Lambda}{v(\mathcal{K}) k^{d}}\left(1-\frac{v(\mathcal{K}) k^{d}}{2 v(\mathcal{K}) \Lambda}\right)^{\lceil\psi \Lambda\rceil} \leq \frac{c_{0} \Lambda}{k^{d}} \mathrm{e}^{-\psi k^{d} / 2}=: \pi_{k},
$$

if $\Lambda$ is large enough that $c_{g}\left(k \Lambda^{-1 / d}\right)^{\gamma_{g}} \leq \frac{1}{2}$. On the complementary event, all of the islands, and thus all of $C$, are covered after an additional time of at most $2 k \lambda_{0}^{-1}$. So take $k=$ $\{(18 / \psi) \log \Lambda\}^{1 / d}$, to make

$$
\pi_{k} \leq \frac{c_{0} \psi}{18 \log \Lambda} \Lambda^{-8}
$$

In a small-world process, we apply Theorem 3.3 instead. With probability $1-O\left(\Lambda^{-\delta}\right)$ for some $\delta>0$, at least $\frac{1}{4} L \rho=\frac{1}{4} \Lambda / d$ ! shortcuts have been encountered by time $\tau(\Lambda, s):=$ $\lambda_{0}^{-1}\left\{\log \Lambda+\tilde{\mathfrak{m}}-\tilde{U}-\log \tilde{C}_{d}\right\}$, where $\tilde{\mathfrak{m}}$ denotes the median $h_{d-1}^{-1}\left(\frac{1}{2}\right)$, since then about half of $C$ has been covered. Because $\mathrm{e}^{\tilde{U}}$ has the distribution of $W_{*}^{[d]}(\infty)$ rather than that of $W_{*}^{[d+1]}(\infty)$, we have $-\tilde{U} \leq(k \log \Lambda)^{1 / d}$, except on an event of probability of order $O\left(\Lambda^{-8}\right)$, if $k$ is chosen large enough, in view of (2.24). The remainder of the argument is as for the gossip process. 


\subsection{Manifolds with boundary}

The assumption that the manifold $C$ is homogeneous simplifies the argument substantially. However, the adjustments needed if $C$ is taken to be a 'reasonable' finite subset of a homogeneous manifold, such as a rectangle in $\mathbb{R}^{2}$ or a spherical cap, are not great. The principal requirement is that most islands do not intersect the boundary $\partial C$. Let $C_{\delta}:=$ $\{P \in C: \mathcal{K}(P, \delta) \cap \partial C \neq \varnothing\}$ denote the $\delta$-neighbourhood of the boundary of $C$, and assume that its volume is not too large when compared with that of $C$ :

$$
L^{-1}\left|C_{\delta}\right| \leq c_{b} \delta\left(\frac{v(\mathcal{K})}{L}\right)^{1 / d}, \quad 0<\delta \leq 2 \log \Lambda,
$$

for some constant $c_{b}$. For instance, with $C$ a square of side $\sqrt{L}$ in $\mathbb{R}^{2}$ and with $\mathcal{K}(P, s)$ a disc of radius $s, d=2$, and $v(\mathcal{K})=\pi, L^{-1}\left|C_{\delta}\right| \leq 4 \delta L^{-1 / 2}$ satisfies (3.20) with $c_{b}=4 / \sqrt{\pi}$. As is clear from the preceding argument, only times less than $\delta_{\Lambda}:=2 \lambda_{0}^{-1} \log \Lambda$ play a significant part, and the probability of an island with randomly chosen centre $P \in C$ intersecting $\partial C$ before time $\delta_{\Lambda}$ is then at most

$$
c_{b} \delta_{\Lambda}\left(\frac{v(\mathcal{K})}{L}\right)^{1 / d}=2 c_{b} \Lambda^{-1 / d} \log \Lambda
$$

under assumption (3.20).

In order to make the arguments of Section 3.3 work, it is enough to be able to bound the growth process above and below by branching processes with constant growth rates $\lambda_{0}^{+}$and $\lambda_{0}^{-}$, respectively, which are close enough to one another, and to have (2.26) hold with an error estimate similar to that in (2.27). For the latter, if (2.25) is satisfied then (3.21) implies that

$$
q_{L}(t, u)=L^{-1} v(\mathcal{K})(t+u)^{d}\left(1+R_{L}(t, u)\right),
$$

where

$$
\left|R_{L}(t, u)\right| \leq c_{g}\left\{(t+u)\left(\frac{v(\mathcal{K})}{L}\right)^{1 / d}\right\}^{\gamma_{g}}+c_{b} \max \{t, u\}\left(\frac{v(\mathcal{K})}{L}\right)^{1 / d},
$$

so that all that is needed is to replace the exponent $\gamma_{g}$ by $\tilde{\gamma}_{g}:=\min \left\{\gamma_{g}, 1\right\}$ and the constant $c_{g}$ by $\tilde{c}_{g}:=c_{g}+c_{b}$ in (2.27) when making intersection calculations. For the former, the upper bound $\lambda_{0}^{+}$given in (3.8) still holds. A lower bound is obtained by neglecting any contacts to points of $C_{\delta_{\Lambda}}$, and taking

$$
\tilde{\lambda}_{0}^{-}:=\lambda_{0}^{-}\left(1-L^{-1}\left|C_{\delta}\right|\right) \geq \lambda_{0}^{-}\left\{1-2 c_{b} \Lambda^{-1 / d} \log \Lambda\right\},
$$

by (3.20) and (3.21). Thus, once again, the previous arguments can be carried through, if, in definition (3.6), $\gamma_{g}$ is replaced by $\tilde{\gamma}_{g}$, and $c_{g}$ by $c_{g}+4 d c_{b} / 3$. This leads to the following result.

Theorem 3.5. For a d-dimensional gossip process on a finite subset $C$ of a homogeneous manifold that satisfies (2.25) and (3.20), the conclusion of Theorem 3.2 holds, but with different constants $a_{1}, a_{2}$, and c. For small-world processes, under the same assumptions, the conclusion of Theorem 3.3 holds, again with different constants.

For the result corresponding to Theorem 3.4, it is also necessary to make the explicit assumption in connection with (3.19), which was previously guaranteed for all $\Lambda$ such that $c_{g}\left(k \Lambda^{-1 / d}\right)^{\gamma_{g}} \leq \frac{1}{2}$, that each of the $n(s)$ sets $\mathcal{K}(P, s)$ used to cover $C$ satisfies

$$
|\mathcal{K}(P, s) \cap C| \geq \frac{1}{2} s^{d} v(\mathcal{K}) .
$$


In the current context, this is no longer automatic, because part of a set $\mathcal{K}(P, s)$ may lie outside $C$. The proof otherwise runs without any change and the conclusion of Theorem 3.4 holds under this extra assumption. Note also that, under the extra assumptions (3.20) and (3.22), the manifold $C$ could also be allowed to consist of a number of disconnected components. In particular, requirement (3.22) applied with $s=k \lambda_{0}^{-1}$ would prevent Theorem 3.4 from being justified if there were components that were too small, and this is to be expected, since it may take an extremely long time for a very small component of $C$ to be hit by a sequence of randomly chosen points of $C$.

\section{Acknowledgements}

ADB thanks the Institute for Mathematical Sciences at the National University of Singapore, and the mathematics departments of the University of Melbourne and Monash University for their kind hospitality while part of the work was undertaken. GDR also thanks the Institute for Mathematical Sciences at the National University of Singapore for their kind hospitality. The authors are grateful to Dirk Schlüter for helpful discussions, and to an anonymous referee whose detailed input has greatly improved the presentation of the paper.

\section{References}

[1] Aldous, D. J. (2013). When knowing early matters: gossip, percolation and Nash equilibria. In Prokhorov and Contemporary Probability Theory (Springer Proc. Math. Statist. 33), eds A. N. Shiryaev, S. R. S. Varadhan and E. L. Presman, Springer, Berlin, pp. 3-27.

[2] Ball, F., Mollison, D. and Scalia-Tomba, G. (1997). Epidemics with two levels of mixing. Ann. Appl. Prob. 7, 46-89.

[3] Ball, F., Sirl, D. and Trapman, P. (2013). Epidemics on random intersection graphs. To appear in Ann. Appl. Prob.

[4] Barbour, A. D. and Reinert, G. (2001). Small worlds. Random Structures Algorithms 19, 54-74. (Correction: 25 (2004), 115.)

[5] Bhamidi, S., van der Hofstad, R. and Hooghiemstra, G. (2012). Universality for first passage percolation on sparse random graphs. Preprint. Available at http://arxiv.org/abs/1210.6839v1.

[6] Chatterjee, S. and Durrett, R. (2011). Asymptotic behavior of Aldous' gossip process. Ann. Appl. Prob. 21, 2447-2482.

[7] McDiarmid, C. (1998). Concentration. In Probabilistic Methods for Algorithmic Discrete Mathematics (Algorithms Combinatorics 16), eds M. Habib et al., Springer, Berlin, pp. 195-248.

[8] Moore, C. and Newman, M. E. J. (2000). Epidemics and percolation in small-world networks. Phys. Rev. E 61, 5678-5682.

[9] Watts, D. J. and Strogatz, S. H. (1998). Collective dynamics of 'small-world' networks. Nature 393, 440442 . 